\title{
Combining multi-omics and drug perturbation profiles to identify novel treatments that improve disease phenotypes in spinal muscular atrophy
}

Katharina E. Meijboom1, 2\#, Viola Volpato1,3\#, Jimena Monzón-Sandoval1,3\#, Joseph M. Hoolachan 4,5, Suzan M. Hammond $1,6,7$, Frank Abendroth8,9, Olivier Gerrit de Jong 1,10, Gareth

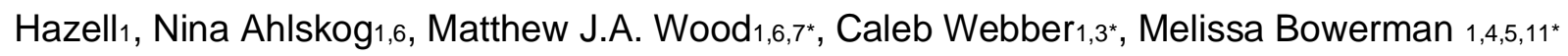

1 Department of Physiology, Anatomy and Genetics, University of Oxford, Oxford, OX1 3QX, United Kingdom

2 Gene Therapy Center, University of Massachusetts Medical School, Worcester, 01605 MA, United States

з UK Dementia Research Institute, Cardiff University, Cardiff, CF24 4HQ, United Kingdom

4 School of Medicine, Keele University, Staffordshire, ST5 5BG, United Kingdom

5 School of Pharmacy and Bioengineering, Keele University, Staffordshire, ST5 5BG, United Kingdom

6 Department of Paediatrics, John Radcliffe Hospital, University of Oxford, Oxford, OX3 9DU, United Kingdom

7 MDUK Oxford Neuromuscular Centre, University of Oxford, OX13QX, United Kingdom 8 Medical Research Council, Laboratory of Molecular Biology, Cambridge, CB2 0QH, United Kingdom

9 Institute of Chemistry, Philipps-University of MarburgHans-Meerwein Strasse 4, D-35032, Marburg, Germany

10 Laboratory Clinical Chemistry \& Hematology, University Medical Center Utrecht, Utrecht University , Heidelberglaan 100, 3584 CX Utrecht, Netherlands

11 Wolfson Centre for Inherited Neuromuscular Disease, RJAH Orthopaedic Hospital, Oswestry, SY10 7AG, United Kingdom

\#: authors contributed equally to the work

*corresponding authors: m.bowerman@keele.ac.uk, WebberC4@cardiff.ac.uk, matthew.wood@paediatrics.ox.ac.uk 


\section{ABSTRACT}

Spinal muscular atrophy (SMA) is a neuromuscular disorder caused by loss of survival motor neuron (SMN) protein. While SMN restoration therapies are beneficial, they are not a cure. We aimed to identify novel treatments to alleviate muscle pathology combining transcriptomics, proteomics and perturbational datasets. This revealed potential drug candidates for repurposing in SMA. One of the lead candidates, harmine, was further investigated in cell and animal models, improving multiple disease phenotypes, including SMN expression and lifespan. Our work highlights the potential of multiple, parallel data driven approaches for development of novel treatments for use in combination with SMN restoration therapies. 


\section{INTRODUCTION}

Spinal muscular atrophy (SMA) is an autosomal recessive neuromuscular disorder 1 and the leading genetic cause of infant mortality 2. The major pathological components of the disease are the selective loss of spinal cord alpha motor neurons, progressive muscle denervation 3 and skeletal muscle atrophy 4 . SMA is caused by mutations in the survival motor neuron 1 (SMN1) gene 5. SMN protein is ubiquitously expressed and complete loss is lethal 6. However, humans have a near-identical centromeric copy of the SMN1 gene, termed SMN2, in which a single nucleotide change (C to $\mathrm{T}$ ) in exon 77 results in the exclusion of exon 7 from $\sim 90 \%$ of the mature transcript 8 . The resulting protein is unstable and gets rapidly degraded 9 . Patients can have a varying number of SMN2 copies, which correlates with disease severity as each SMN2 copy retains the ability to produce $\sim 10 \%$ of functional full-length $(\mathrm{FL})$ protein 10,11 .

The first SMN restoration treatments, Spinraza ${ }^{\mathrm{TM}}$ and Zolgensma $^{\mathrm{TM}}$, have recently been approved by the Food and Drugs Administration (FDA) and the European Medicine Agency (EMA). Spinraza ${ }^{\mathrm{TM}} 12$ is an antisense oligonucleotide (ASO) that promotes SMN2 exon 7 inclusion 13 and is administered by lumbar puncture while Zolgensma ${ }^{\mathrm{TM}}$ delivers SMN1 cDNA via an adeno-associated virus 914 and is administered intravenously. Additional systemically delivered and SMN targeting small molecules are currently being explored in clinical trials such as risdiplam 15,16 . While these treatments have changed the SMA therapeutic landscape, they unfortunately fall short of representing a cure 17-20. There is therefore a present need for SMN-independent therapies that could be used in 
combination with SMN restoration treatments to provide a longer-lasting and more effective therapeutic management of SMA pathology in patients 17-19.

Skeletal muscle pathology is a clear contributor to SMA disease manifestation and progression and improving muscle health could have significant benefits for patients 21 . Here, we used an in-depth, parallel approach combining proteomics, transcriptomics and the drug pertubational dataset Connectivity Map (CMap) 22,23 to identify differentially expressed (DE) genes and proteins in skeletal muscle of the severe Taiwanese Smn--;SMN2 SMA mice 24 that could potentially be restored by known and available pharmacological compounds. This strategy uncovered several potential therapeutic candidates, including harmine, which was further evaluated in cell and animal models, showing an ability to restore molecular networks and improve several disease phenotypes, including SMN expression and lifespan. Our study highlights the tremendous potential of intersecting disease multi-omics with drug perturbational responses to identify therapeutic compounds capable of modulating dysfunctional cellular networks to ameliorate SMA phenotypes. 


\section{RESULTS}

\section{Early restoration of Smn in SMA mice restores muscle protein and transcript expression.}

We first set out to determine the effect of early SMN restoration on the proteomic and transcriptomic profiles of SMA skeletal muscle, with the intent to design therapeutic strategies against the genes and proteins that remain unchanged. To do so, the severe Taiwanese Smn-r;SMN2SMA mouse model 24 received a facial intravenous (IV) injection at post-natal day (P) 0 and P2 of the previously described Pip6a-PMO or Pip6a-scrambled pharmacological compounds $(10 \mu \mathrm{g} / \mathrm{g})$ 25,26. Pip6a is a cell-penetrating peptide (CPP) either conjugated to an SMN2 exon 7 inclusion-promoting ASO (PMO) or a scrambled ASO 25,26. We harvested the tibialis anterior (TA) from P2 (pre-symptomatic) untreated Smn- -;SMN2 and wild type (WT) mice, P7 (symptomatic) untreated Smn- -;SMN2 and WT mice and P7 Pip6a-scrambled- and Pip6a-PMO-treated Smn--;SMN2 mice. TAs were then cut in two, whereby one half was used for transcriptomics and the other for proteomics. qPCR analysis of the ratio of FL SMN2 over total SMN2 confirms a significant increase in FL SMN2 expression in P7 Pip6a-PMO-treated Smn- -;SMN2 mice compared to age-matched untreated and Pip6a-scrambled-treated Smn--;SMN2 mice (Fig. 1a).

Despite differences between transcriptomic and proteomic methodologies highlighted by hierarchical clustering and combined Principal Component Analysis (PCA) (Supplementary Fig. 1), we were able to find clear separation of experimental groups and agreement between transcriptomic and proteomic profiles once the variance attributed to the differences in methodologies was removed (Fig. 1b). At P7, we observed clear separation of Smn--;SMN2- and WT samples, where only P7 Pip6a-PMO treated Smn-^ 
;SMN2- mice clustered with WT. P2 Smn- -;SMN2 and WT samples cluster together suggesting that overt disease cannot be detected in omics readouts at this early stage (Fig. 1b, Supplementary Fig. 2). In the PCA of P7 samples only (Fig. 1c for proteomics and Fig. 1d for transcriptomics), we noted clustering of P7 Pip6a-PMO-treated Smn- - ;SMN2 mice with untreated P7 WT animals, implying full restoration to normal phenotypes. Surprisingly, we also detected segregation of Pip6a-scrambled-treated samples at both transcriptomics and proteomics levels, revealing that presence of the CPP itself impacts transcription and translation (Fig.1 c,d). Importantly, both the combined and separate analysis of transcriptomic and proteomic data allowed us to identify a robust SMA disease signature in muscle and a Pip6a-PMO treatment efficacy signature. Indeed, identification of differentially expressed genes and proteins reveals that early induction of FL SMN expression by Pip6a-PMO normalizes the expression of all transcripts and all but 11 proteins in the TA of Smn- ;SMN2 mice (Table 1, Supplementary Table 1). Of note, one of the proteins that remained significantly downregulated is Smn itself (Supplementary Table 1).

Our in-depth molecular profiling thus demonstrates for the first time that increasing FL SMN2 in neonatal SMA mice quasi-completely normalizes muscle transcripts and proteins, highlighting at the molecular level the potential treatment benefits arising from early intervention.

\section{CMap perturbational profiles identify potential novel non-SMN treatments}

We used the transcriptomic and proteomic profiles of the Smn- - ;SMN2 mice treated with Pip6a-PMO to find drugs that induced similar transcriptional patterns using the 
Connectivity Map (CMap) resource 27,28. For this, we obtained a cleaned and reversed disease signature for both transcriptomics and proteomics data by excluding the genes and proteins restored by Pip6a-scrambled (Pip6a-scrambled-treated Smn-/;SMN2 vs untreated WT) from the overlap between disease (untreated Smn-^;SMN2 vs untreated WT) and Pip6a-PMMO (Pip6a-PMO treated Smn- ;SMN2 vs untreated Smn-r;SMN2) (Fig. 2a). Although these cleaned sets of transcripts and proteins did not show high overlap between omics data types (Fig. 2b), we found similarity at the level of enriched pathways (Fig. 2c). Individual pathway analysis for the disease and the Pip6a-PMO treatment are compiled in Supplementary file 1. The top 10 pharmacological compounds from CMap that showed a reversed pattern of expression for the disease signature, and similar expression patterns to those observed with Pip6a-PMO treatment are listed in Table 2. Importantly, a subset of these drugs, namely salbutamol 29 and alsterpaullone 30 , have already been considered for SMA treatment, highlighting the capability of this analytic approach to identify relevant therapeutic options for SMA.

To further validate our approach, we chose to evaluate the potential of harmine (chemically akin to harmol), a drug identified by its CMap profile but not previously evaluated for SMA, which was present in several proteomic and transcriptomic signatures (Table 2). Harmine is an alkaloid isolated from the seeds of Peganum harmala, traditionally used for ritual and medicinal preparations 31,32. Harmine has also demonstrated therapeutic benefits 33 in animal models of the motor neuron disease amyotrophic lateral sclerosis (ALS) 34 and the muscle disorder myotonic dystrophy type 1 (DM1) 35. 
We firstly validated the genes and proteins predicted to be dysregulated by the transcriptomics and proteomics data and to be normalized by harmine through the CMap analysis. We indeed confirm by qPCR analysis that the genes Snrnp27, Gls, Aspm and Mcm2 are significantly downregulated while Clpx, Ppm1b, Tob2 and Cdkn1a are significantly upregulated in the TA of P7 Smn---;SMN2mice compared to WT animals (Fig. 3a). We then evaluated the ability of harmine to impact the expression of these genes by treating $\mathrm{C} 2 \mathrm{C} 12$ myoblasts, NSC-34 neuronal-like cells, SMA patient fibroblasts and control fibroblasts with 25,35 and $50 \mu \mathrm{M}$ of the drug for 48 hours. We find that harmine demonstrates its predicted activity in a cell- and dose-dependent manner (Fig. 3b). Of note, harmine also displayed inhibitory effects on proliferation and viability at the higher doses in C2C12s and NSC-34s (Supplementary Fig. 3). Finally, we investigated the influence of harmine on SMN expression and observe a significant increased Smn expression in C2C12s and NSC34s at several doses (Fig. 3c). Interestingly, we also find a significant upregulation of FL SMN2 in SMA patient fibroblasts without any changes in total SMN2 (Fig. 3d).

Thus, our strategy of combining transcriptomics, proteomics and drug perturbational datasets has resulted in the generation of a list of several drugs with the potential to restore muscle health in SMA. Importantly, selecting harmine for additional proof-ofconcept investigations, highlights the strength of this approach.

\section{Administration of harmine to SMA mice ameliorates disease phenotypes}

To further evaluate the therapeutic effects of harmine in vivo, we administered it daily to Smn-/-;SMN2 mice and Smn+/-;SMN2 control littermates by gavage (10 mg/kg diluted in 
$0.9 \%$ saline) starting at $\mathrm{P} 0$. We first evaluated the effects of harmine on the expression of Snrnp27, Gls, Aspm, Mcm2, Clpx, Ppm1b, Tob2 and Cdkn1a in muscle (triceps) of P7 untreated and harmine-treated $S m n--; S M N 2$ and $S m n_{+-} ; S M N 2$ mice (Fig. 4a). In vivo, harmine only impacted the expressions of Snrnp27 and Tob2 in Smn-r;SMN2 mice, towards normalized levels (Fig. 4a). Similar to that observed in vitro, harmine administration significantly increased FL SMN2 expression in Smn-r;SMN2 mice but not total SMN2 (Fig. 4b). Total SMN protein levels were also not affected by harmine (Fig. 4c).

We next assessed the effect of harmine upon disease progression and find a significant increase in survival of harmine-treated Smn- ;SMN2 mice compared to untreated Smn-;SMN2 animals (Fig. 4d). Harmine administration also improved weights of treated Smn-;:SMN2 mice compared to untreated Smn- -;SMN2 animals (Fig. 4e). Harmine did not impact the weights of $\mathrm{Smn}_{+/} ; \mathrm{SMN2}$ control littermates (Fig. 4f). An intermediate SMA mouse model, termed Smn2B/-36, was also treated with harmine. Harmine administration to $S m n_{2 B} /$ - mice similarly resulted in a significant increase in survival compared to untreated Smn2B/- animals (Fig. 4g), albeit to a lesser extent, most likely due to the fact that the treated animals developed tremors and needed to be culled. Tremors have indeed been reported in animal studies of long-term harmine administration 37-39. Nevertheless, harmine significantly increased the weights of treated Smn $2 B /-$ mice compared to untreated Smn2B/- animals (Fig. 4h). Interestingly, harmine also had a small but significant impact on the weights of treated $S m n_{2 B /+}$ control littermates compared to untreated Smn2B/+ animals (Fig. 4i). 
Given that harmine was chosen to target molecular effectors in muscle, we measured the myofiber size in the TAs from P7 untreated and harmine-treated Smn--;SMN2 and Smn+/;SMN2 mice. We observe an increased proportion of larger myofibers in harmine-treated Smn- -;SMN2 mice compared to untreated Smn- -;SMN2 animals (Fig. 4j).

Finally, harmine has also been been reported to increase the expression of the neuroprotective glutamate transporter 1 (GLT-1) 40,41 and thus, we assessed GLT-1 protein levels in P7 spinal cords from untreated and harmine-treated Smn- $;$;SMN2 and

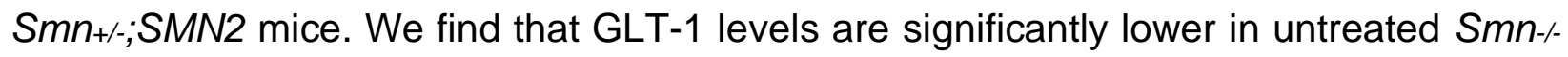
;SMN2 mice compared to untreated $S m n_{+/-} ; S M N 2$ animals and that harmine administration significantly increases GLT-1 expression in treated Smn--;:SMN2 mice (Fig. $4 k)$.

We thus demonstrate that treating SMA mice with harmine significantly improves multiple molecular and pathological phenotypes in both skeletal muscle and the spinal cord.

\section{Harmine administration restores gene expression in muscle of SMA mice}

To systematically explore the effects of harmine in SMA muscle, we performed RNAsequencing (RNA-Seq) on TAs from P7 untreated and harmine-treated Smn--;:SMN2 and WT mice. A total of 15,523 protein coding genes were identified across all samples. We find that harmine significantly reduces the number of differentially expressed genes in Smn--;SMN2 when compared to WT animals (Fig. 5a). Interestingly, harmine treatment in WT animals influences the expression of significantly fewer genes than in Smn-r;SMN2 mice (Fig. 5b). Finally GO analysis with the number of fully (1038) and partially restored (574) genes identifies several pathways that are positively impacted by harmine in SMA 
muscle (Fig. 5c), many of which have been previously implicated in SMA pathology such as glucose metabolism 42.

Our RNA-Seq analysis therefore supports our earlier prediction that harmine could reverse some of the molecular pathologies in SMA muscle.

Harmine restores multiple, but not all, molecular networks disturbed in muscle of Smn---;SMN2 mice

To assess the restorative effects of harmine, we built a gene functional network from the top 500 differentially expressed genes using functional relationships defined by a phenotypic linkage network that links genes together that are likely to influence similar phenotypes 43. Louvain clustering of this network identified six modules of interconnected genes disturbed in Smn--;:SMN2 mice muscle, of which four (M1, M2, M4 and M5) out of six were partially restored by harmine treatment (Fig. 6a). Enrichment analysis in mouse phenotypes highlighted several pathways known to be involved in the pathology such as lipid and glucose metabolism, muscle fiber morphology and contraction (Fig. 6b) providing a molecular explanation for the observed phenotypes in harmine-treated SMA mice and a similarity to the pathways associated with Pip6a-PMO treatment (Fig. 2c). Through Ingenuity Pathway Analysis (IPA), we identified upstream regulators of the restored gene modules (Fig. 6c). The expression of a subset of these upstream regulators was evaluated by qPCR in muscle (triceps) of untreated and harmine-treated Smn-/;SMN2 and $S m n_{+/-;}$SMN2 healthy littermates, based on their relevance to muscle health and SMA pathology. We find that in control $S m n_{+-} ; S M N 2$ control animals, harmine significantly impacted the expression of the dual specificity tyrosine-phosphorylation-regulated kinase 
$1 A($ Dyrk1a) and myogenic differentiation 1 (Myod1) genes (Fig. 7a). In Smn- -;SMN2 mice however, harmine only affected the expression of Myod1 and in the opposite direction (Fig. 7b). To determine if these effects were muscle-specific, we evaluated the expression of Dyrk1a and Myod1 in differentiated WT and siRNA Smn-depleted C2C12 cells that were either untreated or exposed to 25 or $50 \mu \mathrm{M}$ of harmine for 48 hours. Interestingly, we find that harmine significantly reduces Dyrk1a and Myod1 expression in differentiated WT C2C12s without any effect in Smn-depleted cells (Fig. 7c,d), revealing differential in vivo and in vitro effects of harmine. It is therefore evident that additional mechanistic investigations are required to understand the specific and direct benefits of harmine in SMA muscle.

Nevertheless, our bioinformatic analyses uncover several interesting molecular networks restored by harmine in SMA muscle that could have further implications for future development of muscle-specific therapies for SMA. 


\section{DISCUSSION}

Despite the tremendous recent advances in SMA gene therapy, this neuromuscular disorder remains incurable and there is an urgent need for the development of secondgeneration treatments that can be used in combination with $\mathrm{SMN}$-dependent therapies 17-19. In this study, we therefore evaluated and validated a strategy combining transcriptomics, proteomics and drug repositioning to identify novel therapeutic compounds that have the potential to improve muscle pathology in SMA. An in-depth investigation of one of these drugs, harmine, further supports our approach as harmine restored several molecular, behavioural and histological disease phenotypes in both cellular and animal models of the disease.

Of major importance, and to our surprise, we demonstrate that early SMN restoration via Pip6-PMO corrects most, if not all, of the transcriptomic and proteomics dysregulations in SMA muscle, highlighting the need for and likely benefit from early treatment intervention in SMA. It is important to note however that the Pip6a-PMO dose delivered to mice was very high and most likely higher than what would be expected in patients. Our pathway analyses reveal that many molecular functions that are dysregulated in SMA mice compared to WT mice and recovered by Pip6a-PMO have previously been implicated in the pathology of SMA such as RNA metabolism and splicing, circadian regulation of gene expression, ubiquitin pathways, regulation of Rho protein signal transduction and actin binding pathways $44-47$. Their normalization following SMN restoration further supports their involvement in SMA pathology.

Using the differentially expressed genes and protein in SMA muscle compared to WT, we used a CMap pertubational dataset to provide a list of candidate drugs that could improve 
SMA pathology, some of which had previously been evaluated in SMA such as salbutamol 48. CMap analysis has previously been used to identify new potential therapeutics for a range of different conditions such as skeletal muscle atrophy 49 , osteoarthritic pain 50, osteoporosis 51, gliomas 52, lung adenocarcinoma 53, hepatoblastoma 54, acute myeloid leukemia 55 and kidney disease 56 . CMap can also help establish prediction models for different adverse drug reactions and to evaluate drug safety 57.

In this study, we chose to provide a more in-depth assessment of harmine, a drug predicted to restore differentially expressed genes and proteins in SMA muscle. Interestingly, one of the genes downregulated in SMA muscle and restored by harmine is Snrnp27, a small nuclear RNP (snRNP) involved in pre-mRNA splicing 58 and SMN plays a canonical role in the assembly of snRNPs 59. Cyclin dependent kinase inhibitor $1 \mathrm{~A}$ (Cdkn1a or p21) was also identified as a potential molecular target of harmine. This mediator of cell cycle and DNA repair is reported to be upregulated in various SMA models $60-64$. In addition to specific genes, GO analysis of our RNA-Seq data reveals that harmine restores several genes implicated in key muscle processes such as muscle structure development, muscle contraction, muscle system process and muscle cell differentiation. Thus, our combined transcriptomics, proteomics and CMap analysis has identified genes that have previously been implicated in SMA pathology.

While harmine was selected as a non-SMN treatment strategy, we found an upregulation of FL SMN2 but not total SMN2 in SMA cells and mice, implying that harmine possibly affects alternative splicing of SMN2. Interestingly, harmine restored the alternative splicing of TNNT2 and INSR in DM1 myoblasts and muscle from DM1 mice 35, further 
supporting a role for harmine in modulating mRNA splicing. As demonstrated by our RNASeq analysis, harmine further restores the expression of several genes, indicating that its potential benefits may stem from combinatorial effects on SMN and non-SMN genes. The latter most likely makes the greatest contribution to the therapeutic benefits observed, given that harmine did not increase SMN protein levels.

In addition, harmine can cross the blood-brain barrier and has well characterized neuroprotective properties, including its ability to upregulate the expression of GLT-1 in several neurodegenerative models 40,41 . We indeed show that GLT-1 expression is reduced in the spinal cord of SMA mice and significantly upregulated following harmine administration. Reduced glutamate transporter activity throughout the CNS of SMA patients has also been reported 65 . The fact that harmine exerts muscle and CNS effects makes it an interesting therapeutic option for SMA. However, it is important to note that harmine can also exert adverse effects such as the onset of tremors 37-39, which we observed when dosing the intermediate Smn2B/- mouse model over a longer period of time.

To our knowledge, this is the first in-depth validation of this combinatorial approach in SMA. We are able to show strength and potential of combining multi-omics and drug repositioning to uncover novel therapeutic entities, which in this case was aimed at improving muscle health in SMA. Our work thus provides an invaluable list of pharmacological compounds that can be evaluated for treatment of SMA muscle pathology as well as strong support for the use of this combined multi-omics and bioinformatic strategy. 


\section{ONLINE METHODS}

\section{Animals and animal procedures}

Wild-type mice (FVB/N 66 and C57BL/6J 67) were obtained from Jackson Laboratories. The severe Smn-/,;SMN2+/- mouse model 68 was also obtained from Jackson Laboratories (FVB.Cg-Smn1tm1Hung Tg(SMN2)2Hung/J). The moderate Smn2B/- mouse model 69 was generously provided by Dr. Lyndsay M Murray, University of Edinburgh). All experiments with live animals were performed at the Biomedical Services Building, University of Oxford. Experimental procedures were authorized and approved by the University of Oxford ethics committee and UK Home Office (current project license PDFEDC6F0, previous project license 30/2907) in accordance with the Animals (Scientific Procedures) Act 1986.

The Pip6a-PMO and Pip6a-scrambled conjugates were both separately prepared in $0.9 \%$ saline solution and administered at a dose of $10 \mu \mathrm{g} / \mathrm{g}$ via an intravenous facial vein injection at $\mathrm{PO}$ and $\mathrm{P} 2$.

Harmine hydrochloride (sc-295136, Insight Biotechnology Ltd, Sante Cruz) was dissolved in $0.9 \%$ saline and administered daily $(10 \mathrm{mg} / \mathrm{kg}$ ) by gavage.

\section{Synthesis of Pip6a peptide-PMO conjugates}

The PMO sequence targeting ISS-N1 intron 7 (-10-27) (5'-ATTCACTTTCATAATGCTGG3') and scrambled PMO (5'-TAC GTT ATA TCT CGT GAT AC-3') were purchased from Gene Tools LLC (Corvallis).

The Pip6a Ac-(RXRRBRRXRYQFLIRXRBRXRB)-COOH peptide was manufactured by standard 9-fluorenylmethoxy carbonyl chemistry, purified to $>90 \%$ purity by reverse- 
phase high-performance liquid chromatography (HPLC) and conjugated to the 3' end of the PMO through an amide linkage. The conjugate was purified by cation exchange HPLC, desalted and analyzed by mass spectrometry. Pip6a peptide-PMO conjugates were dissolved in sterile water and filtered through a $0.22 \mu \mathrm{m}$ cellulose acetate membrane before use.

\section{Laminin staining of skeletal muscle}

Tibialis anterior (TA) muscles were fixed in 4\% PFA overnight. Tissues were sectioned $(13 \mu \mathrm{m})$ and incubated in blocking buffer for 2 hours $(0.3 \%$ Triton-X, 20\% FBS and 20\% normal goat serum in PBS). After blocking, tissues were stained overnight at $4{ }^{\circ} \mathrm{C}$ with rat anti-laminin (Sigma) in blocking buffer. The next day, tissues were washed in PBS and probed using goat-anti-rat IgG 488 secondary antibodies (Invitrogen) for one hour. PBSwashed tissues were mounted in Fluoromount-G (Southern Biotech). Images were taken with a DM IRB microscope (Leica) with a 20x objective. Quantitative assays were performed blinded on 3-5 mice for each group and five sections per mouse. The area of muscle fiber within designated regions of the TA muscle sections was measured using Fiji 70.

\section{qPCR}

RNA was extracted from tissues and cells by either an RNeasy kit from Qiagen or by guanidinium thiocyantate-acid-phenol-chloroform extraction using TRIzol Reagent (Life Technologies) as per manufacturer's instructions. The same RNA extraction method was employed for similar experiments and equal RNA amounts were used between samples 
within the same experiments. cDNA was prepared with the High Capacity cDNA Kit (Life Technologies) according to the manufacturer's instructions. The cDNA template was amplified on a StepOnePlus Real-Time PCR Thermocycler (Life Technologies) with SYBR Green Mastermix from Applied Biosystems. qPCR data was analyzed using the StepOne Software v2.3 (Applied Biosystems). Primers used for qPCR were obtained from IDT and sequences for primers were either self-designed or ready-made (Supplementary Table 2). Relative gene expression was quantified using the Pfaffl method 71 and primer efficiencies were calculated with the LinRegPCR software. We normalized relative expression level of all tested genes in mouse tissue and cells to RNA polymerase II polypeptide $\mathrm{J}($ PolJ) 72 . For human cells, we ran a GeNorm kit (Primer Design) to identify RPL13A as a reference/housekeeping gene. Primers for RPL13A were from IDT (209604333).

\section{Cell culture}

Both C2C12s 73 and NSC-34s 74 cell lines were maintained in growth media consisting of Dulbecco's Modified Eagle's Media (DMEM) supplemented with $10 \%$ fetal bovine serum (FBS) and 1\% Penicillin/Streptomycin (all Life Technologies). The cells were cultured at $37^{\circ} \mathrm{C}$ with $5 \% \mathrm{CO}_{2} . \mathrm{C} 2 \mathrm{C} 12$ myoblasts were differentiated in DMEM containing $2 \%$ horse serum (HS) for 7 days to form multinucleated myotubes.

For siRNA experiments, C2C12 cells were seeded in 12-well plates and after reaching $50 \%$ confluence, growth media was changed to differentiation media and the cells were transfected with $10 \mu \mathrm{M}$ of siSmn (Duplex name: mm.RiSmn1.13.1) and scrambled siRNA (scrambled negative control DsiRNA, \#51-01-19-08) (both from IDT) in an siRNA- 
lipofectamine complex (Lipofectamine® RNAiMAX Reagent, Life Technologies). Fresh media containing the transfection reagents was changed every 2 days. At $D 6$, the $\mathrm{C} 2 \mathrm{C} 12$ myotubes were further exposed to harmine $(25$ and $50 \mu \mathrm{M})$ for 48 hours.

Human fibroblasts were obtained from Coriell Institue (SMA GM03813, control AG02261) and cultured in DMEM, supplemented with $1 \%$ antibiotics/antimycotics and $20 \%$ FBS.

\section{MTS assays}

Cell viability and proliferation of $\mathrm{C} 2 \mathrm{C} 12$ and NSC-34 cells treated with harmine (sc202644, Insight Biotechnology Ltd, Sante Cruz) dissolved in DMSO (final concentration $0.03 \%)$ were evaluated with a 3-(4,5-dimethylthiazol-2-yl)-5-(3-carboxymethoxyphenyl)2-(4-sulfophenyl)-2H-tetrazolium (MTS) assay kit (Colorimetric). The measurements were made according to manufacturer's instructions. Briefly, $10 \mu \mathrm{l}$ of MTS reagent was added directly to the wells and cell plates were incubated at $37^{\circ} \mathrm{C}$ for a minimum of 1 hour. Absorbance was measured at $490 \mathrm{~nm}$ on a CLARIOstar ${ }^{\circledR}$ plate reader (BMG LABTECH). Background absorbance was first subtracted using a set of wells containing medium only, then normalized to and expressed as a relative percentage of the plate-averaged untreated control. To chemically induce apoptosis, cells were treated with $10 \mu \mathrm{M}$ Staurosporine (Abcam, Cambridge, UK).

\section{Western blot}

Freshly prepared radioimmunoprecipitation (RIPA) buffer was used to homogenize tissue and cells, consisting of $50 \mathrm{mM}$ Tris $\mathrm{pH} 8.8,150 \mathrm{mM} \mathrm{NaCl}, 1 \% \mathrm{NP}-40,0.5 \%$ Sodium Deoxycholate, $0.1 \%$ SDS and complete mini-proteinase inhibitors (1 tablet per $10 \mathrm{ml}$ 
extraction solution, Roche). Equal amounts of total protein were loaded, as measured by Bradford Assay. Protein samples were first diluted 1:1 with Laemmli sample buffer (BioRad, Hemel Hempstead, UK) containing 5\% $\beta$-mercaptoethanol (Sigma) and heated at $100^{\circ} \mathrm{C}$ for 10 minutes. Next, samples were loaded on freshly made $1.5 \mathrm{~mm} 12 \%$ polyacrylamide separating and $5 \%$ stacking gel and electrophoresis was performed at $120 \mathrm{~V}$ for $\sim 1.5 \mathrm{~h}$ in running buffer. Subsequently, proteins were transferred from the gel onto to a polyvinylidene fluoride (PVDF) membrane (Merck Millipore) via electroblotting at $120 \mathrm{~V}$ for 60 minutes in transfer buffer containing $20 \%$ methanol. Membranes were then incubated for $2 \mathrm{~h}$ in Odyssey Blocking Buffer (Licor). The membrane was then probed overnight at $4^{\circ} \mathrm{C}$ with primary antibodies (anti-GLT-1, 1:1000, Abcam \#ab41621; antiSMN, 1:1000, Millipore \#MABE230; anti-vinculin, 1:200.000, Sigma \#V9131) in Odyssey Blocking Buffer and 0.1\% Tween-20. The next day, after three 10-minute washing steps with PBS, the membrane was incubated for 1 hour at room temperature with secondary antibodies conjugated to infrared dyes. Lastly, the membrane was washed again three times 10 minutes in PBS and visualized by scanning $700 \mathrm{~nm}$ and $800 \mathrm{~nm}$ channels on the LI-COR Odyssey CLx infrared imaging system (LI-COR) for 2.5 minutes per channel. The background was subtracted and signal of protein of interest was divided by signal of the housekeeping protein or total protein, per sample.

\section{Proteomic analysis}

Proteomic analyses were performed using a liquid chromatography-mass spectrometry (LC-MS)-based method. High-resolution isoelectric focusing (HiRIEF) was used at the peptide level in the $3.7-5.0 \mathrm{pH}$ range. Two tandem mass tags (TMTs, chemical labels) 
were used for mass spectrometry (MS)-based quantification and identification of proteins.

The data was median normalized based on peptide ratio. Amongst a total of 9798 potentially detectable proteins, most (8152) were identified in all samples/groups.

The limma R package was used for differential expression analysis, whereby differentially expressed proteins were defined by FDR $<0.05$. Gene Ontology enrichment analysis of proteomic data was executed using topGO R function and adjusted $p$ values for multiple testing following a Benjamini-Hochberg correction. For principal component analysis, we used the prcomp $\mathrm{R}$ function on the normalized expression data.

\section{Microarray analysis}

RNA was extracted by guanidinium thiocyantate-acid-phenol-chloroform extraction using TRIzol Reagent (Life Technologies) as per manufacturer's instructions. GeneChip Mouse Transcriptome Assay 1.0 arrays were used (Affymetrix core facility, Karolinska Institute) with $100 \mathrm{ng}$ of RNA per sample. Annotations for the Mouse Transcript Array 1.0 at the transcript level were obtained from the Affymetrix website (http://www.affymetrix.com/products_services/arrays/specific/mo_trans_assay.

affx\#1_4). We performed background correction and RMA normalization at the probe level using oligo $\mathrm{R}$ package. We summarized the data in ensemble transcript IDs using the average. The total number of ensemble transcript IDs was 93,594, corresponding to 37,450 genes. For differential expression analysis, we used limma $R$ package and considered a transcript differentially expressed if their FDR $<0.05$. A gene was considered differentially expressed if at least one of the associated transcripts was differentially expressed. Gene Ontology enrichment analysis was performed in R using 
the topGO function as described for proteomic data. For principal component analysis we used the prcomp R function on the RMA normalized gene expression data at the gene level (for comparison with proteomic data).

\section{Combined analysis of proteomic and transcriptomic data}

To measure the similarity between gene expression profiles, we used the Ward hierarchical clustering on the Euclidean distance of $1-r$ (where $r$ is the Pearson correlation between samples). To compare the two omics readouts, proteomic and transcriptomic data were scaled (transformed to z-score values), followed by a PCA analysis showing that PC1 divides the data at the transcript and protein level (Supplementary Fig. 1). Using the kill.pc function in the swamp R package, we extracted a new expression matrix where the variance given by PC1 has been removed. Finally, we performed hierarchical clustering analysis on the new expression matrix.

\section{RNA-Sequencing analysis}

RNA was extracted using a RNeasy Microarray Tissue Mini Kit from Qiagen. Lysis and homogenization were performed using QIAzol Lysis Reagent. cDNA synthesis and RNASeq library construction were performed at the Oxford Genomics Centre (Oxford, United Kingdom) using poly(A) enrichment of the mRNA (mRNA-Seq) and HiSeq 4000 Systems for sequencing. All samples passed quality control. For differential expression analysis, we used DESeq2 on genes expressed across all samples (15523 genes) after removal of one outlier (Harmine-treated SMA sample 1). We considered a gene differentially expressed at FDR $<0.05$. For Gene Ontology enrichment analysis, we used topGO R 
function and adjusted $p$ values for multiple testing following a Benjamini-Hochberg correction. For mouse phenotype enrichment analysis, we downloaded phenotypes from the Mouse Genome Database (MGD), Mouse Genome Informatics, The Jackson Laboratory, Bar Harbor, Maine (URL: http://www.informatics.jax.org) (June, 2018) and used in-house script to correct for the background set of expressed genes.

\section{Gene functional network and clustering method}

A gene functional network is built by extracting interactions from a phenotypic linkage network 43 for the top 500 differentially expressed genes between WT and SMA samples. To identify modules of highly interconnected genes in the network, we employed "cluster_louvain" function in "igraph" R package 75. This function implements the multilevel modularity optimization algorithm 76,77 where at each step genes are re-assigned to modules in a greedy way and the process stops when the modularity does not increase in a successive step.

\section{Upstream regulators}

Ingenuity Pathway Analysis (www.qiagenbioinformatics.com) was used to identify the top 100 upstream regulators for the top 500 differentially expressed genes_between WT and SMA samples. A reduced list of regulators was identified based on their target genes to be within the four harmine-reversed modules.

\section{CMap analysis}

Ensembl transcript ids from mice were mapped to human probe IDs (HG-U133A) using biomaRt (Ensembl transcript id mus musculus $\rightarrow$ Ensembl gene id mus musculus $\rightarrow$ 
ortholog_one2one $\rightarrow$ Ensembl gene id homo sapiens $\rightarrow$ HG-U133A id). We compared the identified disease and Pip6a-PMO signatures (top 500 up-regulated and top 500 down-regulated genes/proteins) to the drug instances contained in the CMap dataset (Build 02, http://www.broadinstitute.org/cmap), which are defined as the basic unit of data and metadata in CMap.

\section{Statistical Analysis}

All statistical analyses were done with the most up-to-date Graphpad Prism software. When appropriate, a Student's unpaired two-tail $t$-test, a one-way ANOVA followed by a Tukey's multiple comparison test or a two-way ANOVA followed by a Sidak's multiple comparison test was used. Outliers were identified via the Grubbs' test. For the KaplanMeier survival analysis, the log-rank test was used and survival curves were considered significantly different at $p<0.05$. 


\section{FIGURE LEGENDS}

Figure 1. Restoration of protein and transcript expression in skeletal muscle of SMA mice following early SMN restoration treatment. Smn- ; SMN2 mice received a facial intravenous injection at postnatal day (P) 0 and P2 of Pip6a-scrambled or Pip6aPMO $(10 \mu \mathrm{g} / \mathrm{g})$. The tibialis anterior was harvested from P2 untreated Smn-r;:SMN2 and WT mice, P7 untreated, Pip6a-scrambled-treated and Pip6a-PMO-treated Smn--;:SMN2 mice and P7 untreated WT mice. a. Comparison of the ratio of full length (FL) SMN2 over total SMN2 quantified by qPCR between P7 untreated Pip6a-scrambled- and Pip6aPMO-treated Smn- -;SMN2 mice. Data are mean \pm s.d., $\mathrm{n}=4$ animals per experimental group, one-way ANOVA, ns $=$ not significant, ${ }^{* * *} p<0.001$. b. Heatmap shows the similarity between transcriptomic and proteomic expression profiles measured by the Pearson correlation between each pair of samples (after the removal of the first principal component). c. First two principal components constructed from proteomic profiles mice discriminate Smn-/-;SMN2 mice from WT at P7. Notably, only mice treated with Pip6aPMO cluster along with WT mice. d. First two principal components based on transcriptional profiles of P7 mice show similar clustering of Pip6a-PMO cluster and WT mice.

Figure 2. Identification of disease signal reversed by treatment with Pip6a at both proteomics and transcriptomics level. a. We retained the proteins (left) and genes (right) that were differentially expressed between untreated Smn- ;SMN2 and WT (disease), reversed by treatment with Pip6a and not differentially expressed between scrambled-Pip6a treated Smn--;:SMN2 and WT. We called these as cleaned signatures. 
b. Overlap of the cleaned signatures between proteins and genes. Although the overlap was not high, we detected higher overlaps for genes and proteins with the same directionality (both up or both down). c. Enriched GO Biological Processes terms that showed similarity across comparisons. The greatest similarity between genes and proteins was detected for the cleaned signatures.

Figure. 3. Harmine, as predicted by CMap analyses, is able to reverse the expression of genes differentially expressed in SMA muscle in several cellular models. a. qPCR analysis of genes predicted to be significantly downregulated (Snrnp27, Gls, Aspm and Mcm2) and upregulated (Clpx, Ppm1b, Tob2 and Cdkn1a) in the TA of untreated P7 SMA Smn--;SMN2 and WT mice. Data are mean \pm s.d., $\mathrm{n}=4$ animals per experimental group, $t$ test, $p=0.041$ (Snrnp27), $p=0.0019$ (G/s), $p=0.0001$ (Aspm), $p<0.0001$ (Mcm2), $p<0.0001$ (Clpx), $p=0.0076$ (Ppm1b), $p=0.0012$ (Tob2), $p<0.0001$ (Cdkn1a). b. C2C12s, NSC34s, SMA fibroblasts and control fibroblasts were treated with 25, 35 of $50 \mu \mathrm{M}$ of harmine for 48 hours. Expression of Snrnp27, Gls, Aspm, Mcm2, Clpx, Ppm1b, Tob2 and Cdkn1a was assessed by qPCR and compared to untreated cells. Data are mean \pm s.d., $\mathrm{n}=3$ independent wells, two-way ANOVA, ${ }^{*} p<0.05,{ }^{* *} p<0.01$ ${ }^{* * *} p<0.001,{ }^{* * * *} p<0.0001$. c. C2C12s and NSC34s were treated with 25, 35 of $50 \mu \mathrm{M}$ of harmine for 48 hours. Expression of Smn was assessed by qPCR and compared to untreated cells. Data are mean \pm s.d., $n=3$ independent wells, one-way ANOVA, ${ }^{\star} p<0.05$ ${ }^{* *} p<0.01,{ }^{* * * *} p<0.0001$. d. SMA fibroblasts were treated with 25,35 of $50 \mu \mathrm{M}$ of harmine for 48 hours. Expression of FL SMN2 and total SMN2 was assessed by qPCR and 
compared to untreated cells. Data are mean \pm s.d., $n=3$ independent wells, one-way ANOVA, ns = not significant, ${ }^{*} p<0.05$

Figure 4. Administration of harmine to SMA mice ameliorates several disease phenotypes. All treated animals received a daily dose of harmine $(10 \mathrm{mg} / \mathrm{kg}$, diluted in 0.9\% saline) by gavage starting at postnatal day (P) 0. a. qPCR analysis of Snrnp27, Gls, Aspm, Mcm2, Clpx, Ppm1b, Tob2 and Cdkn1a in triceps of P7 untreated and harminetreated Smn-/-;SMN2 mice and Smn+/-;SMN2 control littermates. Data are mean \pm s.d., $\mathrm{n}$ = 4 animals per experimental group except for harmine-treated Smn+/;SMN2 where $\mathrm{n}=$ 3, two-way ANOVA, * $p<0.05$. b. qPCR analysis of FL SMN2 and total SMN2 in triceps of P7 untreated and harmine-treated Smn-/-;SMN2 mice and Smn+/-;SMN2 control littermates. Data are mean \pm s.d., $n=4$ animals per experimental group except for harmine-treated $S m n_{+/-} ; S M N 2$ where $n=3$, two-way ANOVA, * $p<0.05$. c. Western blot analysis of SMN protein in triceps of P7 untreated Smn+/-;SMN2 control littermates and P7 harmine-treated and untreated Smn-/;SMN2 mice and. Data are mean \pm s.d., $\mathrm{n}=3$ animals per experimental group, two-way ANOVA, ${ }^{* *} p<0.01$. d. Survival curves of untreated and harmine-treated Smn-/,SMN2 mice. Data are Kaplan Meier survival curve, $\mathrm{n}=10$ for untreated Smn-/;SMN2 mice, $\mathrm{n}=11$ for harmine-treated Smn-/-;SMN2 mice, Log-rank (Mantel-Cox) test, ${ }^{*} p=0.0211$. e. Daily weights of untreated and harminetreated Smn-/,;SMN2 mice. Data are mean \pm s.d., $\mathrm{n}=10$ for untreated Smn-/;SMN2 mice, $\mathrm{n}=11$ for harmine-treated Smn- - SMN2 mice, two-way ANOVA, ${ }^{* * *} p<0.001$ ${ }^{* * * *} p<0.0001$. f. Daily weights of untreated and harmine-treated $S m n_{+/-;} ; M N 2$ mice. Data are mean \pm s.d., $\mathrm{n}=13$ for untreated $S m n_{+/-} ; S M N 2$ mice, $\mathrm{n}=15$ for harmine-treated 
Smn+/-;SMN2 mice, two-way ANOVA. g. Survival curves of untreated and harminetreated Smn2B/- mice. Data are Kaplan Meier survival curve, $\mathrm{n}=9$ for untreated Smn2B/mice, $\mathrm{n}=7$ for harmine-treated Smn2B/- mice, Log-rank (Mantel-Cox) test, ${ }^{*} p=0.0221$. h. Daily weights of untreated and harmine-treated $S m n 2 B /-$ mice. Data are mean \pm s.d., $\mathrm{n}=$ 9 for untreated Smn2B/- mice, $\mathrm{n}=7$ for harmine-treated Smn2B/- mice, two-way ANOVA, ${ }^{*} p<0.05,{ }^{* *} p<0.01,{ }^{* * *} p<0.0001$. i. Daily weights of untreated and harmine-treated Smn2B/+ mice. Data are mean \pm s.d., $\mathrm{n}=13$ for untreated $S m n 2 B /+$ mice, $\mathrm{n}=8$ for harminetreated Smn2B/- mice, two-way ANOVA, ${ }^{*} p<0.05,{ }^{* \star} p<0.01$. j. Relative frequency of myofiber sizes in P7 untreated and harmine-treated Smn-/-;SMN2 and Smn+/-;SMN2 mice. Data are percentages, $n=3$ animals per experimental group and $>400$ myofibers per experimental group. k. Representative western blots and quantification of Glt-1/vinculin expression in the spinal cord of P7 untreated and harmine-treated Smn--;SMN2 and $S m n_{+/-}$;SMN2 mice. Data are mean \pm s.d., $\mathrm{n}=3$ for untreated and harmine-treated $S m n_{+/-}$ ;SMN2 mice, $\mathrm{n}=4$ for untreated and harmine-treated Smn-^-;SMN2 mice, two-way ANOVA, ${ }^{* *} p<0.01,{ }^{* * *} p<0.001$

Figure 5. RNA sequencing and pathway analysis reveals full rescue of $20 \%$ of dysregulated genes in SMA muscle following harmine administration. All treated animals received a daily dose of harmine $(10 \mathrm{mg} / \mathrm{kg}$, diluted in $0.9 \%$ saline $)$ by gavage starting at postnatal day $(P) 0$. TAs were harvested at P7 from untreated and harminetreated Smn-/,;SMN2 mice and WT animals and processed for RNA sequencing. a. Venn diagram representation of the differentially expressed genes based on the negative binomial distribution (DESeq2) in untreated Smn--;SMN2 mice vs WT mice, harmine- 
treated Smn--;SMN2 mice vs WT mice and untreated Smn-/;SMN2 mice vs harminetreated Smn-/;SMN2 mice. b. Venn diagram representation of the differentially expressed genes based on the negative binomial distribution (DESeq2) in untreated Smn-/;SMN2 mice vs WT mice, harmine-treated Smn-^-SMN2 mice vs WT mice and untreated WT mice vs harmine-treated WT mice. c. Gene Ontology (GO)/molecular function for fully and partly restored genes in muscle of harmine-treated Smn-/;SMN2 mice.

Figure 6. Identification of molecular effectors involved in harmine activity in SMA muscle. a. Gene functional network built on the top 500 differentially expressed genes, colored by WT vs SMA (left) and by SMA vs harmine-treated (right). b. Top MGI enriched phenotypes for the six identified modules in the network in a. c. Proportions of target genes within each of the six modules that are predicted to be regulated by the identified upstream regulators by IPA.

Figure 7. Differential in vivo and in vitro effects of harmine on muscle expression of predicted molecular effectors. All treated animals received a daily dose of harmine (10 mg/kg, diluted in $0.9 \%$ saline) by gavage starting at postnatal day (P) 0 . a. qPCR analysis of Dyrk1A, Sirt1, Pgc1 $\alpha$, MyoD1, Pten, Rora and GR $\alpha$ expression in triceps of P7 untreated and harmine-treated $S m n_{+/-;} S M N 2$ control littermates. Data are mean \pm s.d., $\mathrm{n}=4$ animals for untreated $S m n_{+/} ; S M N 2$ mice and 3 for harmine-treated $S m n_{+/} ; S M N 2$, two-way ANOVA, ${ }^{*} p<0.05$. b. qPCR analysis of Dyrk1A, Sirt1, Pgc1 $\alpha$, MyoD1, Pten, Rora and GR $\alpha$ expression in triceps of P7 untreated and harmine-treated Smn-r;SMN2 mice. Data are mean \pm s.d., $\mathrm{n}=3$ animals for untreated $S m n--; S M N 2$ mice and 5 for harmine- 
treated Smn--;SMN2, two-way ANOVA, ${ }^{* *} p<0.001$. c. qPCR analysis $D y r k 1 A$ expression in differentiated WT and Smn-depleted C2C12s that were untreated or exposed to 25 or $50 \mu \mathrm{M}$ harmine. Data are mean \pm s.d., $\mathrm{n}=3$ for each experimental group, two-way ANOVA, ${ }^{*} p<0.05,{ }^{* *} p<0.01$, ns $=$ not significant. d. $q P C R$ analysis MyoD1 expression in differentiated WT and Smn-depleted C2C12s that were untreated or exposed to 25 or 50 $\mu \mathrm{M}$ harmine. Data are mean \pm s.d., $\mathrm{n}=3$ for each experimental group, two-way ANOVA, ${ }^{* *} p<0.01,{ }^{* *} p<0.001$, ns $=$ not significant.

\section{TABLES}

Table 1. Number of differentially expressed (DE) transcripts and proteins between experimental groups.

Table 2. Top 10 top pharmacological compounds identified by CMap analysis based on three expression signatures for both the transcriptomic data and proteomic data.

\section{SUPPLEMENTARY FIGURE LEGENDS}

Supplementary Figure 1. Clustering and principal component analysis integrating proteomic and transcriptomic data. a. Heatmap correspond to the Pearson correlation between each pair of proteomic and transcriptomic profiles. Gene expression profiles show higher correlations than proteomic data, but similar clustering of experimental groups is observed within each type of data. b. Principal component analysis of the proteomic and transcriptomic data separates by data by type (RNA or Protein) along the first component. 
Supplementary Figure 2. Principal component analysis on proteomic (a) and transcriptomic (b) data of untreated post-natal day (P) 2 and P7 Smn--;SMN2 and WT mice.

Supplementary Figure 3. In vitro Dose-dependent toxicity of harmine treatment in vitro. C2C12s (a) and NSC-34s (b) were treated with 1, 10, 25 or $50 \mu \mathrm{M}$ for 24,48 or 72 hours. Control groups were untreated cells or cells treated with either DMSO (vehicle) or Staurosporine (positive control). An MTS assay was performed on all experimental groups and MTS scores are normalized to untreated cells at 24 hours (100\%). Data are mean \pm s.d., $\mathrm{n}=3$ independent wells, two-way ANOVA, ${ }^{* *} p<0.01,{ }^{* \star *} p<0.001$ ${ }^{* * * *} p<0.0001$.

\section{SUPPLEMENTARY TABLES}

Supplementary Table 1. Proteins downregulated in P7 Pip6a-PMO-treated Smn--;:SMN2 mice compared to P7 untreated WT mice.

Supplementary Table 2. List of mouse and human qPCR primers.

\section{SUPPLEMENTARY FILES}

Supplementary File 1. Top biological processes enriched among the genes differentially expressed in disease and Pip6a-PMO treatment. 


\section{ACKNOWLEDGEMENTS}

We would like to thank the staff at the BMS facility at the University of Oxford, Dr Emelie Blomberg and Dr Samir El-Andaloussi (Karolinska Institute) for the microarray services, Dr Henrik Johansson (Karolinska Institute) for the proteomic services and the Oxford Genomics Centres for the RNA Sequencing services. K.E.M. was funded by the MDUK and SMA Trust. M.B. was funded by the SMA Trust. J.M.H. is funded by the Keele University School of Medicine. S.M.H. is funded by the MRC DPFS (MR/R025312/1). Computation used the Oxford Biomedical Research Computing (BMRC) facility, a joint development between the Wellcome Centre for Human Genetics and the Big Data Institute supported by Health Data Research UK and the NIHR Oxford Biomedical Research Centre. The views expressed are those of the author(s) and not necessarily those of the NHS, the NIHR or the Department of Health. 


\section{REFERENCES}

1. Ben-Shachar, S., Orr-Urtreger, A., Bardugo, E., Shomrat, R. \& Yaron, Y. Large-scale population screening for spinal muscular atrophy: Clinical implications. Genetics in Medicine 13, 110-114 (2011).

2. Miniño, A. M., Xu, J. \& Kochanek, K. D. National Vital Statistics Reports, Volume 59, Number 2, (December 9, 2010). (2008).

3. Wadman, R. I., Vrancken, A. F. J. E., van den Berg, L. H. \& van der Pol, W. L. Dysfunction of the neuromuscular junction in spinal muscular atrophy types 2 and 3. Neurology 79, 2050-5 (2012).

4. Crawford, T. O. \& Pardo, C. A. The neurobiology of childhood spinal muscular atrophy. Neurobiology of disease 3, 97-110 (1996).

5. Lefebvre, S. et al. Identification and Characterization of a Spinal Muscular AtrophyDetermining Gene. Cell 80, 155-65 (1995).

6. Schrank, B. et al. Inactivation of the survival motor neuron gene, a candidate gene for human spinal muscular atrophy, leads to massive cell death in early mouse embryos. Proceedings of the National Academy of Sciences of the United States of America 94, 9920-5 (1997).

7. Lorson, C. L., Hahnen, E., Androphy, E. J. \& Wirth, B. A single nucleotide in the SMN gene regulates splicing and is responsible for spinal muscular atrophy. Proceedings of the National Academy of Sciences 96, 6307-6311 (1999).

8. Monani, U. R. A single nucleotide difference that alters splicing patterns distinguishes the SMA gene SMN1 from the copy gene SMN2. Human Molecular Genetics 8, 1177-1183 (1999).

9. Chang, H.-C., Hung, W.-C., Chuang, Y.-J. \& Jong, Y.-J. Degradation of survival motor neuron $(\mathrm{SMN})$ protein is mediated via the ubiquitin/proteasome pathway. Neurochemistry International 45, 1107-1112 (2004). 
10. Butchbach, M. E. R. Copy Number Variations in the Survival Motor Neuron Genes: Implications for Spinal Muscular Atrophy and Other Neurodegenerative Diseases. Front Mol Biosci 3, 7 (2016).

11. Lefebvre, S. et al. Correlation between severity and SMN protein level in spinal muscular atrophy. Nat. Genet. 16, 265-269 (1997).

12. Hoy, S. M. Nusinersen: First Global Approval. Drugs 77, 473-479 (2017).

13. Hua, Y., Vickers, T. A., Okunola, H. L., Bennett, C. F. \& Krainer, A. R. Antisense masking of an hnRNP A1/A2 intronic splicing silencer corrects SMN2 splicing in transgenic mice. American journal of human genetics 82, 834-48 (2008).

14. Zolgensma - one-time gene therapy for spinal muscular atrophy. Med Lett Drugs Ther 61, 113-114 (2019).

15. Shorrock, H. K., Gillingwater, T. H. \& Groen, E. J. N. Overview of Current Drugs and Molecules in Development for Spinal Muscular Atrophy Therapy. Drugs 78, 293-305 (2018).

16. Ratni, H. et al. Discovery of Risdiplam, a Selective Survival of Motor Neuron-2 ( SMN2) Gene Splicing Modifier for the Treatment of Spinal Muscular Atrophy (SMA). J. Med. Chem. 61, $6501-6517$ (2018).

17. Gidaro, T. \& Servais, L. Nusinersen treatment of spinal muscular atrophy: current knowledge and existing gaps. Developmental Medicine \& Child Neurology 61, 19-24 (2019).

18. Wood, M. J. A., Talbot, K. \& Bowerman, M. Spinal muscular atrophy: antisense oligonucleotide therapy opens the door to an integrated therapeutic landscape. Hum. Mol. Genet. 26, R151-R159 (2017).

19. Bowerman, M. et al. Therapeutic strategies for spinal muscular atrophy: SMN and beyond. Dis Model Mech 10, 943-954 (2017).

20. Ramos, D. M. et al. Age-dependent SMN expression in disease-relevant tissue and implications for SMA treatment. J. Clin. Invest. 129, 4817-4831 (2019). 
21. Boyer, J. G., Ferrier, A. \& Kothary, R. More than a bystander: the contributions of intrinsic skeletal muscle defects in motor neuron diseases. Front Physiol 4, 356 (2013).

22. Lamb, J. et al. The Connectivity Map: Using Gene-Expression Signatures to Connect Small Molecules, Genes, and Disease. Science vol. 313 1929-1935 (2006).

23. Segal, M. R., Xiong, H., Bengtsson, H., Bourgon, R. \& Gentleman, R. Querying Genomic Databases: Refining the Connectivity Map. Statistical Applications in Genetics and Molecular Biology 11, (2012).

24. Hsieh-Li, H. M. et al. A mouse model for spinal muscular atrophy. Nat. Genet. 24, 66-70 (2000).

25. Hammond, S. M. et al. Systemic peptide-mediated oligonucleotide therapy improves longterm survival in spinal muscular atrophy. Proceedings of the National Academy of Sciences 113, 10962-10967 (2016).

26. Walter, L. M. et al. Interventions Targeting Glucocorticoid-Krüppel-like Factor 15-BranchedChain Amino Acid Signaling Improve Disease Phenotypes in Spinal Muscular Atrophy Mice. EBioMedicine 31, 226-242 (2018).

27. Lamb, J. et al. The Connectivity Map: using gene-expression signatures to connect small molecules, genes, and disease. Science 313, 1929-1935 (2006).

28. Subramanian, A. et al. A Next Generation Connectivity Map: L1000 Platform and the First 1,000,000 Profiles. Cell 171, 1437-1452.e17 (2017).

29. Pane, M. et al. Daily salbutamol in young patients with SMA type II. Neuromuscular Disorders $18,536-540$ (2008).

30. Makhortova, N. R. et al. A screen for regulators of survival of motor neuron protein levels. Nature Chemical Biology 7, 544-552 (2011).

31. Niroumand, M. C., Farzaei, M. H. \& Amin, G. Medicinal properties of Peganum harmala L. in traditional Iranian medicine and modern phytotherapy: a review. Journal of Traditional Chinese Medicine 35, 104-109 (2015). 
32. dos Santos, R. G. \& Hallak, J. E. C. Effects of the Natural $\beta$-Carboline Alkaloid Harmine, a Main Constituent of Ayahuasca, in Memory and in the Hippocampus: A Systematic Literature Review of Preclinical Studies. Journal of Psychoactive Drugs 49, 1-10 (2017).

33. Moloudizargari, M., Mikaili, P., Aghajanshakeri, S., Asghari, M. H. \& Shayegh, J. Pharmacological and therapeutic effects of Peganum harmala and its main alkaloids. Pharmacognosy reviews 7, 199-212 (2013).

34. Foran, E. \& Trotti, D. Glutamate transporters and the excitotoxic path to motor neuron degeneration in amyotrophic lateral sclerosis. Antioxidants \& redox signaling 11, 1587-602 (2009).

35. Herrendorff, R. et al. Identification of Plant-derived Alkaloids with Therapeutic Potential for Myotonic Dystrophy Type I. The Journal of biological chemistry 291, 17165-77 (2016).

36. Bowerman, M., Murray, L. M., Beauvais, A., Pinheiro, B. \& Kothary, R. A critical smn threshold in mice dictates onset of an intermediate spinal muscular atrophy phenotype associated with a distinct neuromuscular junction pathology. Neuromuscul. Disord. 22, 263-276 (2012).

37. Coates, G. H. \& Cox, B. Harmine tremor after brain monoamine oxidase inhibition in the mouse. European journal of pharmacology 18, 284-6 (1972).

38. AHMED, A. \& TAYLOR, N. R. The analysis of drug-induced tremor in mice. British journal of pharmacology and chemotherapy 14, 350-4 (1959).

39. Kulkarni, S. K. \& Kaul, P. N. Modification by levo-propranolol of tremors induced by harmine in mice. Experientia 35, 1627-1628 (1979).

40. Li, Y. et al. Harmine, a natural beta-carboline alkaloid, upregulates astroglial glutamate transporter expression. Neuropharmacology 60, 1168-75 (2011).

41. Sun, P., Zhang, S., Li, Y. \& Wang, L. Harmine mediated neuroprotection via evaluation of glutamate transporter 1 in a rat model of global cerebral ischemia. Neuroscience Letters 583, 32-36 (2014). 
42. Bowerman, M. et al. Glucose metabolism and pancreatic defects in spinal muscular atrophy. Ann. Neurol. 72, 256-268 (2012).

43. Honti, F., Meader, S. \& Webber, C. Unbiased functional clustering of gene variants with a phenotypic-linkage network. PLoS Comput. Biol. 10, e1003815 (2014).

44. Groen, E. J. N. \& Gillingwater, T. H. UBA1: At the Crossroads of Ubiquitin Homeostasis and Neurodegeneration. Trends Mol Med 21, 622-632 (2015).

45. Bowerman, M., Shafey, D. \& Kothary, R. Smn depletion alters profilin II expression and leads to upregulation of the RhoA/ROCK pathway and defects in neuronal integrity. J. Mol. Neurosci. 32, 120-131 (2007).

46. Walter, L. M. et al. Light modulation ameliorates expression of circadian genes and disease progression in spinal muscular atrophy mice. Hum. Mol. Genet. (2018) doi:10.1093/hmg/ddy249.

47. Singh, R. N., Howell, M. D., Ottesen, E. W. \& Singh, N. N. Diverse role of survival motor neuron protein. Biochim Biophys Acta Gene Regul Mech 1860, 299-315 (2017).

48. Tiziano, F. D. et al. Longitudinal evaluation of SMN levels as biomarker for spinal muscular atrophy: results of a phase Ilb double-blind study of salbutamol. J. Med. Genet. (2018) doi:10.1136/jmedgenet-2018-105482.

49. Kunkel, S. D. et al. mRNA expression signatures of human skeletal muscle atrophy identify a natural compound that increases muscle mass. Cell metabolism 13, 627-38 (2011).

50. Chang, M., Smith, S., Thorpe, A., Barratt, M. J. \& Karim, F. Evaluation of Phenoxybenzamine in the CFA Model of Pain following Gene Expression Studies and Connectivity Mapping. Molecular Pain 6, 1744-8069-6-56 (2010).

51. Brum, A. M. et al. Connectivity Map-based discovery of parbendazole reveals targetable human osteogenic pathway. Proceedings of the National Academy of Sciences of the United States of America 112, 12711-6 (2015). 
52. Koh, L. W.-H. et al. A Distinct Reactive Oxygen Species Profile Confers Chemoresistance in Glioma-Propagating Cells and Associates with Patient Survival Outcome. Antioxidants \& Redox Signaling 19, 2261-2279 (2013).

53. Wang, G. et al. Expression-Based In Silico Screening of Candidate Therapeutic Compounds for Lung Adenocarcinoma. PLoS ONE 6, e14573 (2011).

54. Beck, A. et al. Connectivity map identifies HDAC inhibition as a treatment option of high-risk hepatoblastoma. Cancer Biology \& Therapy 17, 1168-1176 (2016).

55. Manzotti, G. et al. Monocyte-macrophage differentiation of acute myeloid leukemia cell lines by small molecules identified through interrogation of the Connectivity Map database. Cell cycle (Georgetown, Tex.) 14, 2578-89 (2015).

56. Zhong, Y. et al. Renoprotective Effect of Combined Inhibition of Angiotensin-Converting Enzyme and Histone Deacetylase. Journal of the American Society of Nephrology 24, 801811 (2013).

57. Wang, K. et al. Systematic drug safety evaluation based on public genomic expression (Connectivity Map) data: Myocardial and infectious adverse reactions as application cases. Biochemical and Biophysical Research Communications 457, 249-255 (2015).

58. Wahl, M. C., Will, C. L. \& Lührmann, R. The Spliceosome: Design Principles of a Dynamic RNP Machine. Cell 136, 701-718 (2009).

59. Pellizzoni, L., Yong, J. \& Dreyfuss, G. Essential role for the SMN complex in the specificity of snRNP assembly. Science (New York, N.Y.) 298, 1775-9 (2002).

60. Jangi, M. et al. SMN deficiency in severe models of spinal muscular atrophy causes widespread intron retention and DNA damage. Proceedings of the National Academy of Sciences 114, E2347-E2356 (2017).

61. Staropoli, J. F. et al. Rescue of gene-expression changes in an induced mouse model of spinal muscular atrophy by an antisense oligonucleotide that promotes inclusion of SMN2 exon 7. Genomics 105, 220-228 (2015). 
62. Zhang, Z. et al. SMN Deficiency Causes Tissue-Specific Perturbations in the Repertoire of snRNAs and Widespread Defects in Splicing. Cell 133, 585-600 (2008).

63. Bäumer, D. et al. Alternative splicing events are a late feature of pathology in a mouse model of spinal muscular atrophy. PLoS Genetics 5, e1000773 (2009).

64. Ruggiu, M. et al. A role for SMN exon 7 splicing in the selective vulnerability of motor neurons in spinal muscular atrophy. Molecular and cellular biology 32, 126-38 (2012).

65. Hayashi, M. et al. Oxidative stress and disturbed glutamate transport in spinal muscular atrophy. Brain Dev. 24, 770-775 (2002).

66. Taketo, M. et al. FVB/N: an inbred mouse strain preferable for transgenic analyses. Proceedings of the National Academy of Sciences of the United States of America 88, 20659 (1991).

67. Mekada, K. et al. Genetic differences among C57BL/6 substrains. Experimental animals 58, 141-9 (2009).

68. Hsieh-Li, H. M. et al. A mouse model for spinal muscular atrophy. Nature genetics 24, 66-70 (2000).

69. Hammond, S. M. et al. Mouse survival motor neuron alleles that mimic SMN2 splicing and are inducible rescue embryonic lethality early in development but not late. PloS one 5, e15887 (2010).

70. Schindelin, J. et al. Fiji: an open-source platform for biological-image analysis. Nature Methods 9, 676-682 (2012).

71. Pfaffl, M. W. A new mathematical model for relative quantification in real-time RT-PCR. Nucleic Acids Res. 29, e45 (2001).

72. Radonić, A. et al. Guideline to reference gene selection for quantitative real-time PCR. Biochem. Biophys. Res. Commun. 313, 856-862 (2004).

73. YAFFE, D. \& SAXEL, O. Serial passaging and differentiation of myogenic cells isolated from dystrophic mouse muscle. Nature 270, 725-727 (1977). 
74. Cashman, N. R. et al. Neuroblastoma $\times$ spinal cord (NSC) hybrid cell lines resemble developing motor neurons. Developmental Dynamics 194, 209-221 (1992).

75. Csardi, G. \& Nepusz, T. The igraph software package for complex network research. InterJournal Complex Systems, 1695 (2006).

76. Lambiotte, R., Delvenne, J.-C. \& Barahona, M. Random Walks, Markov Processes and the Multiscale Modular Organization of Complex Networks. IEEE Transactions on Network Science and Engineering 1, 76-90 (2014).

77. Blondel, V. D., Guillaume, J.-L., Lambiotte, R. \& Lefebvre, E. Fast unfolding of communities in large networks. J. Stat. Mech. 2008, P10008 (2008). 
bioRxiv preprint doi: https://doi.org/10.1101/2019.12.17.879353; this version posted December 18, 2019. The copyright holder for this preprint (which was not certified by peer review) is the author/funder, who has granted bioRxiv a license to display the preprint in perpetuity. It is made available under aCC-BY 4.0 International license.

a

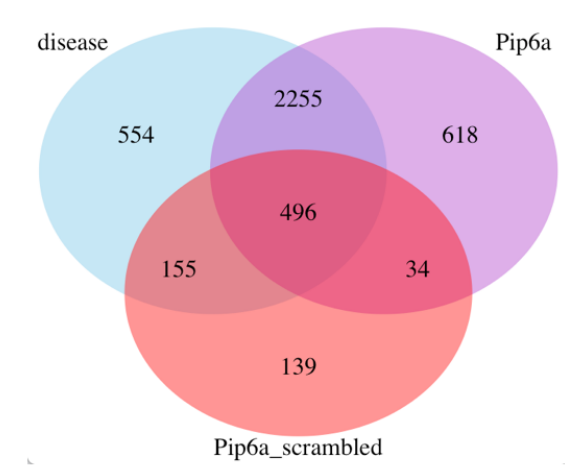

Genes

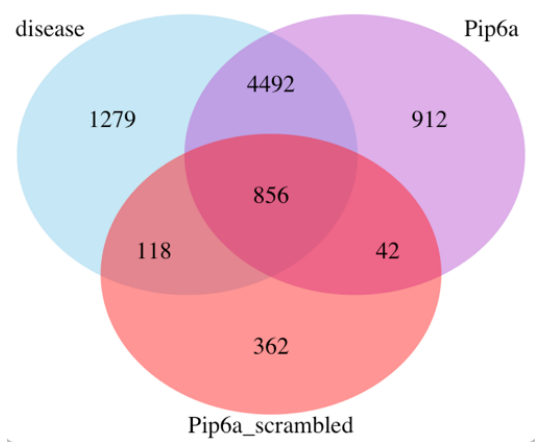

overlapClean_down_P overlapClean_up_P

b overlapClean_down_G

1109 0

0

0

90

0

19

112

\section{C}

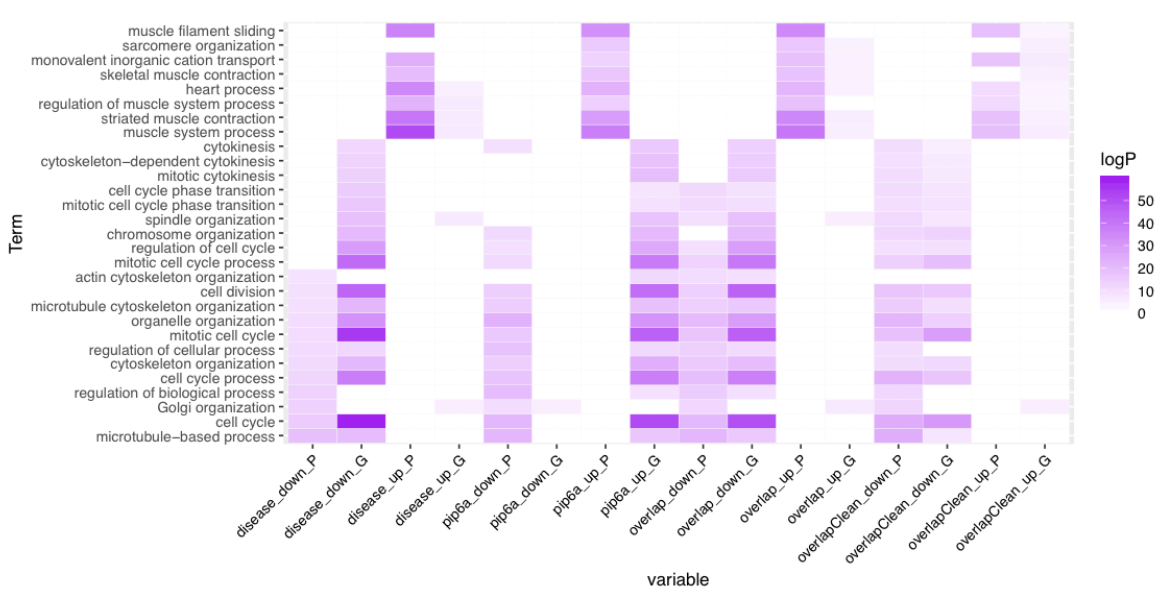




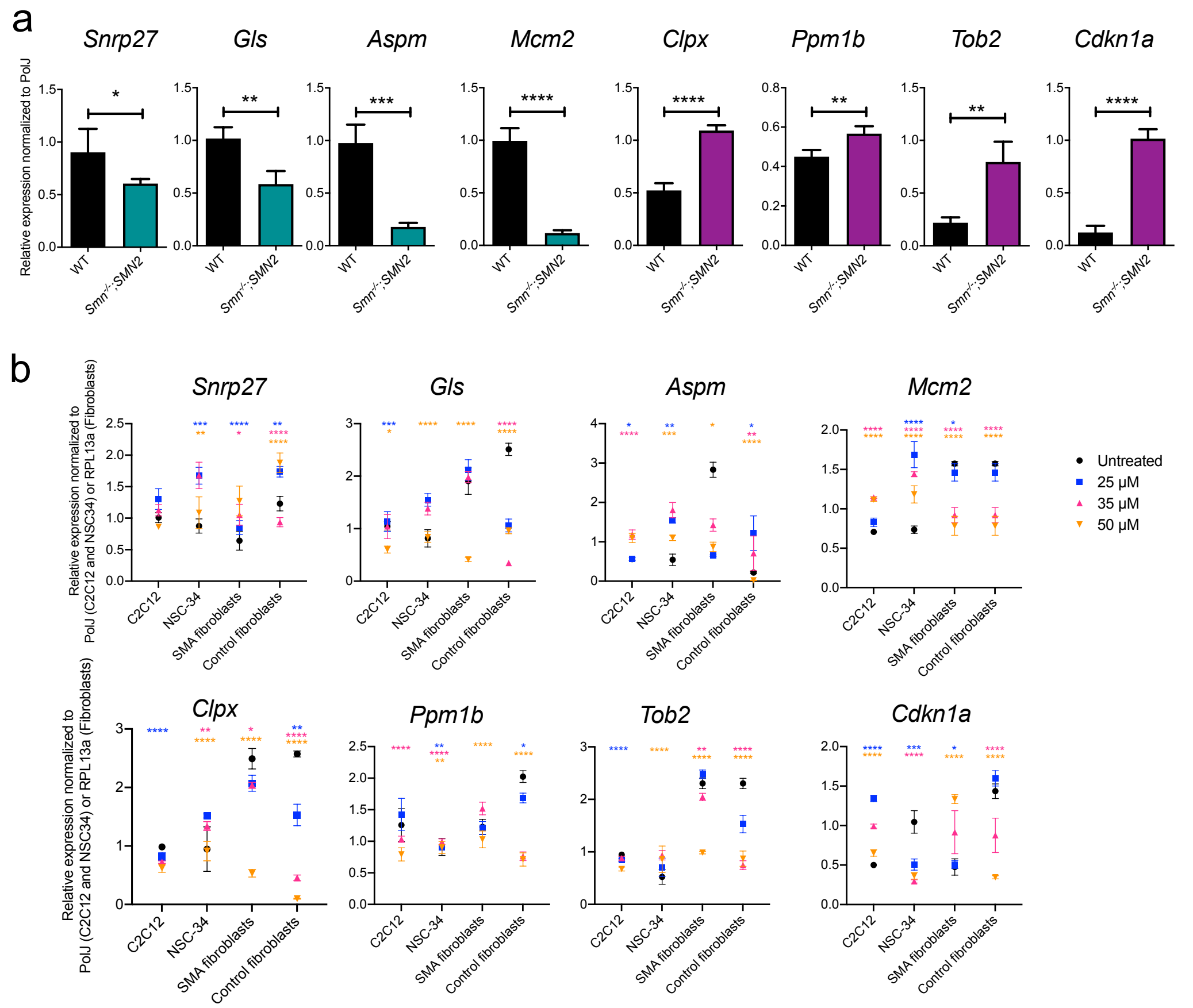

C Smn_C2C12

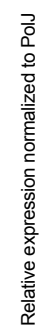

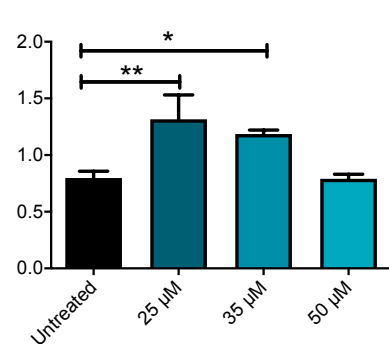

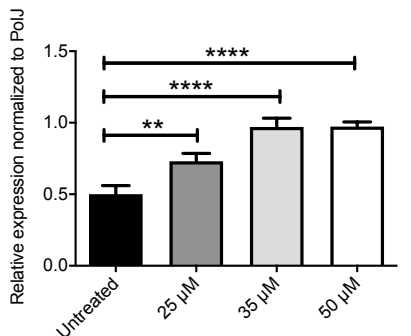

Smn_NSC-34

d
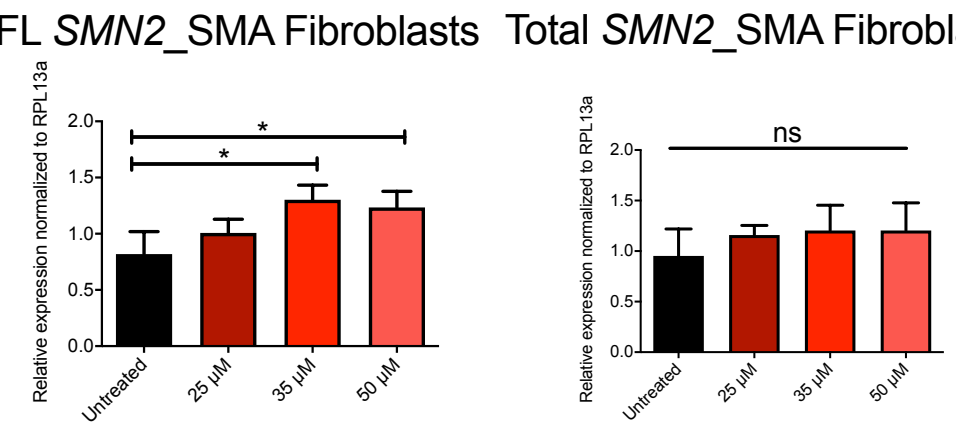
(which was not certified by peer review) is the author/funder, who has granted bioRxiv a license to display the preprint in perpetuity. It is made Untreated available under aCC-BY 4.0 International license.

$\square$ Harmine $(10 \mathrm{mg} / \mathrm{kg})$

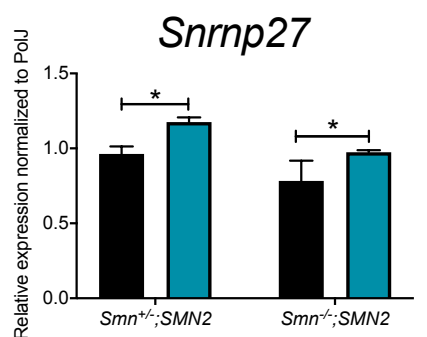

Clpx

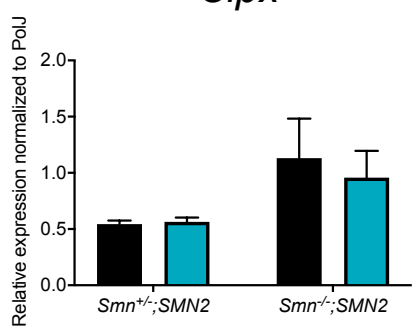

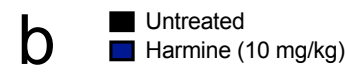

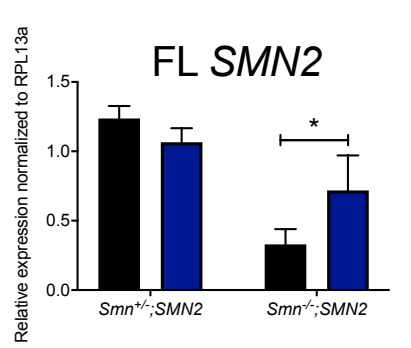

d
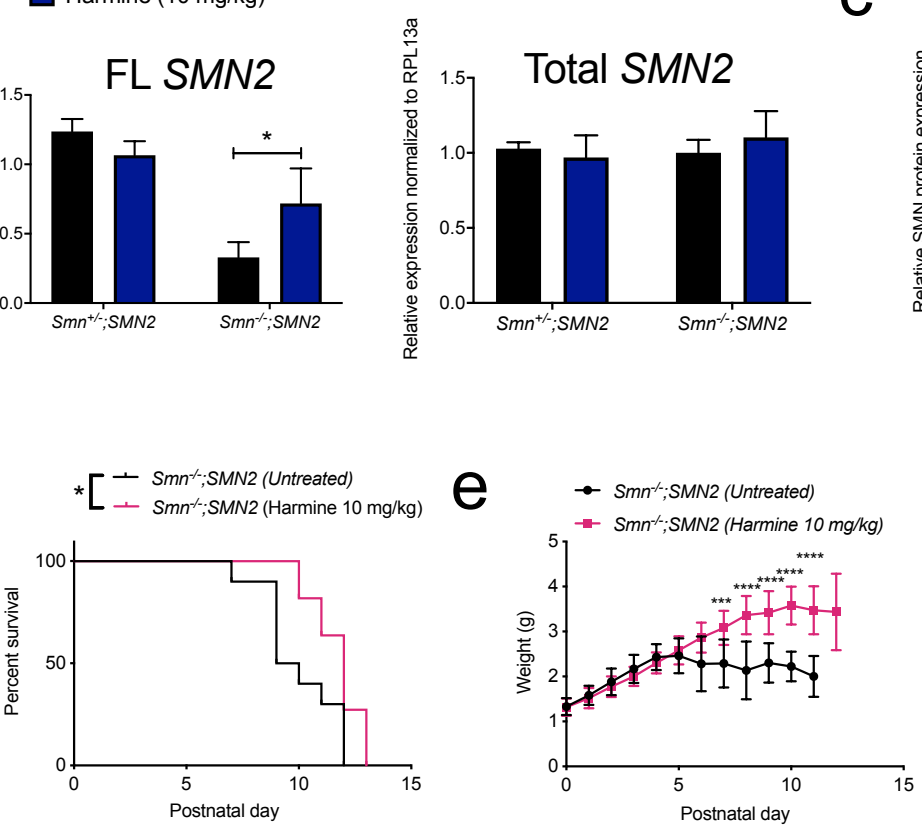

C
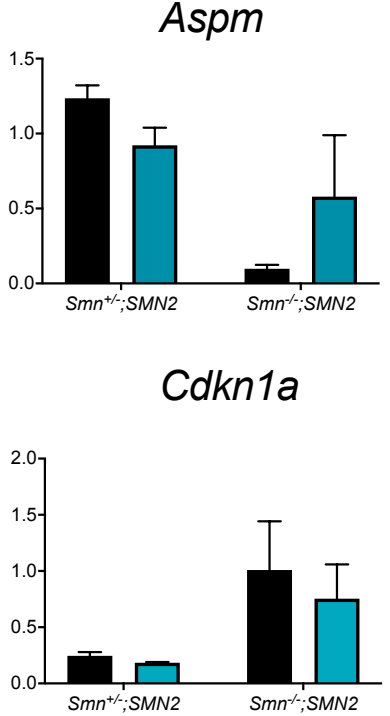

Cdkn1a
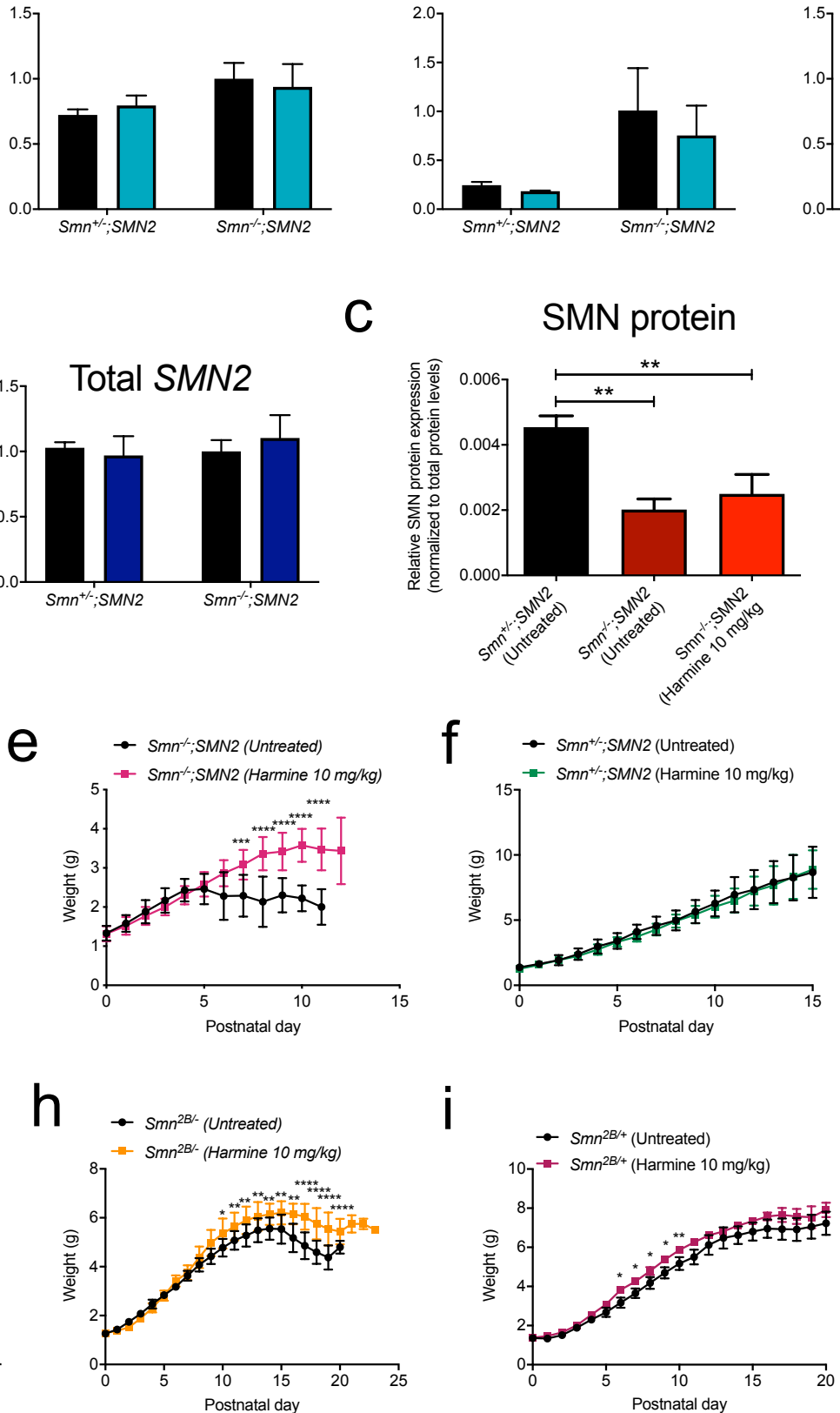

g

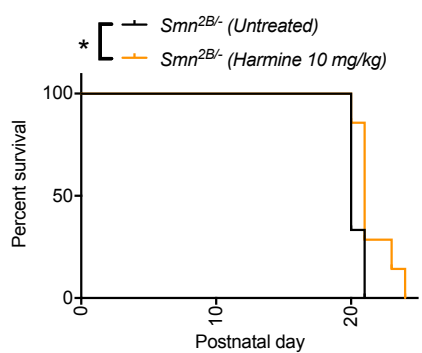

Mcm2

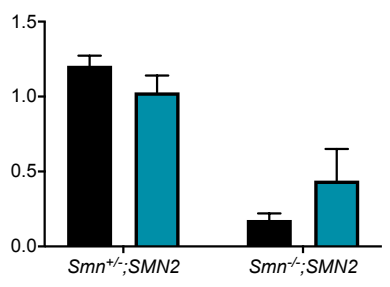

Tob2

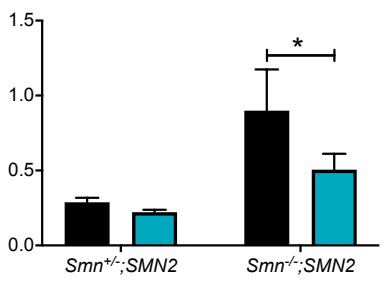

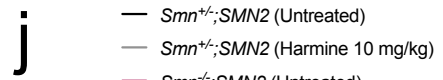

- Smn ${ }^{-} ;$SMN2 (Untreated)

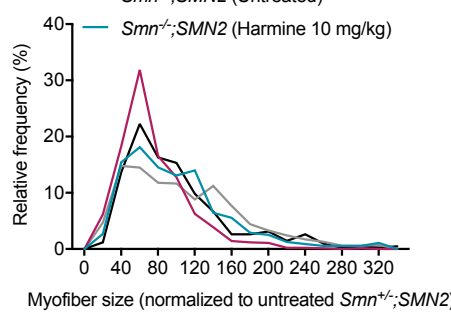

k

Vinculin

Glt-1
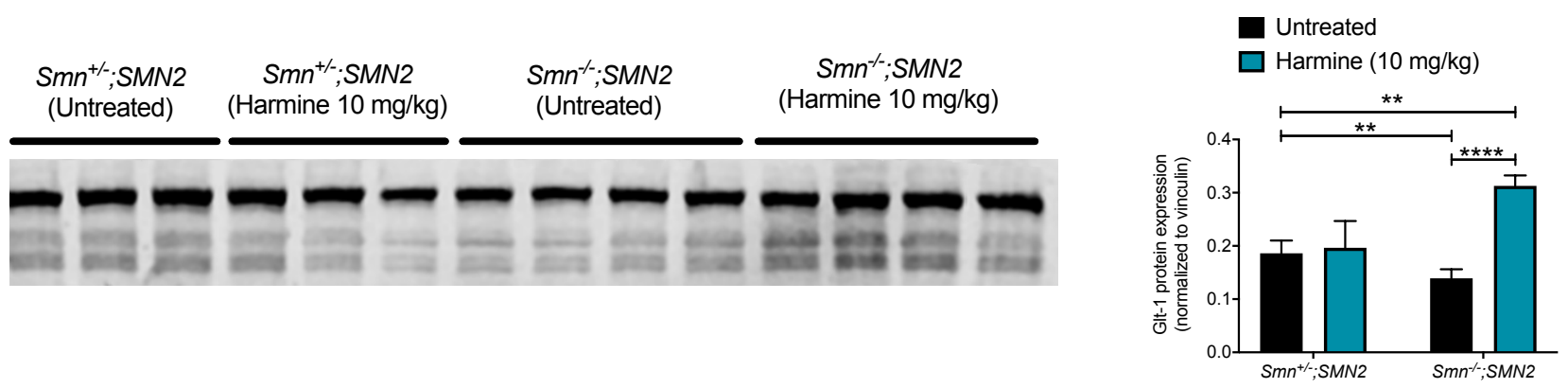
Untreated SMA

vs

Untreated WT

5183

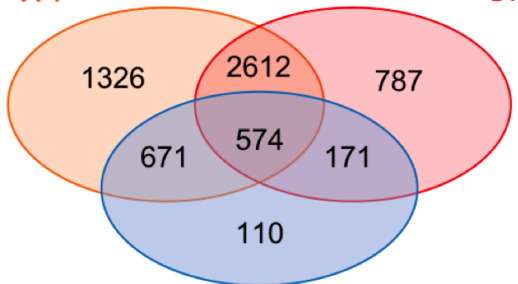

Harmine-treated SMA

vs

Untreated SMA

1426
Untreated SMA

vs

Untreated WT

5183

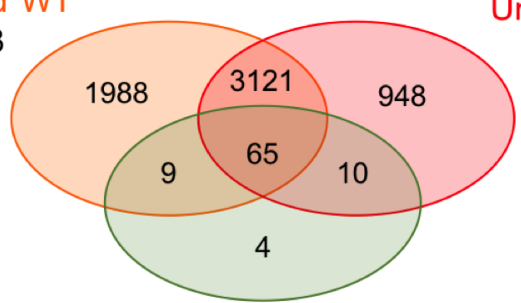

Harmine-treated WT

vs

Untreated WT

88

C

circulatory system development muscle structure development small molecule catabolic process oxidation-reduction process regulation of cellular carbohydrate meta...

collagen fibril organization fatty acid oxidation muscle cell differentiation regulation of glucose metabolic process muscle contraction monocarboxylic acid catabolic process fatty acid catabolic process lipid oxidation -

supramolecular fiber organization system process fatty acid beta-oxidation muscle system process striated muscle cell differentiation extracellular structure organization extracellular matrix organization purine ribonucleoside triphosphate metab. ribonucleoside triphosphate metabolic pr... nucleoside triphosphate metabolic proces... ribonucleotide metabolic process

purine ribonucleotide metabolic process purine nucleoside triphosphate metabolic... purine-containing compound metabolic pro...

purine nucleotide metabolic process ATP metabolic process purine ribonucleoside monophosphate meta.. ribonucleoside monophosphate metabolic p... purine nucleoside monophosphate metaboli... nucleoside monophosphate metabolic proce... amide biosynthetic process

peptide biosynthetic process peptide metabolic process organonitrogen compound biosynthetic pro... cellular amide metabolic process cytoplasmic translation -

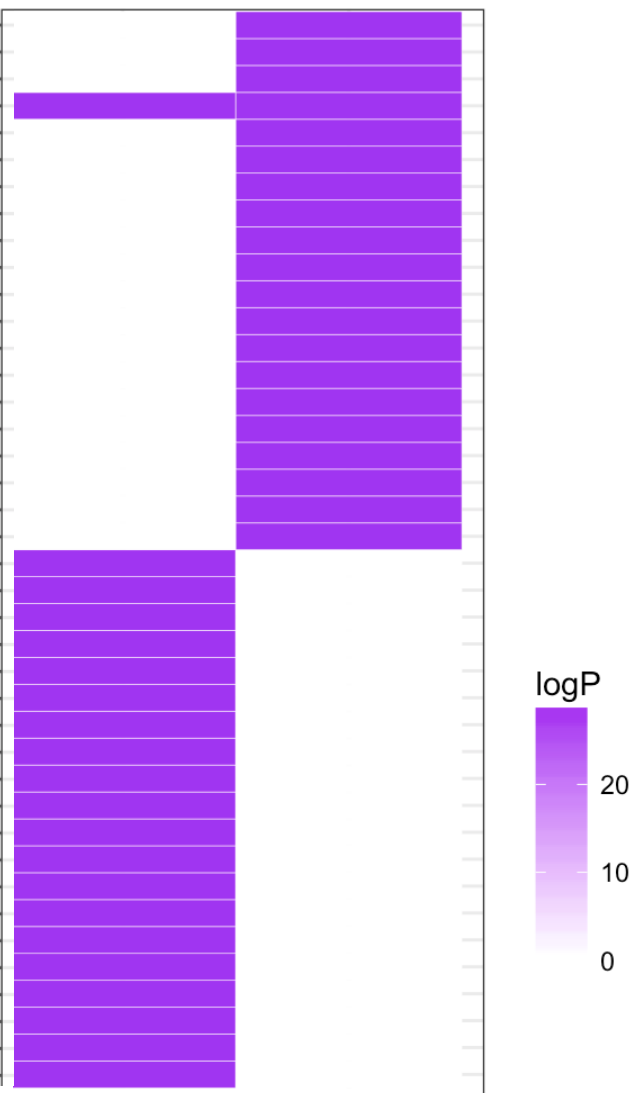

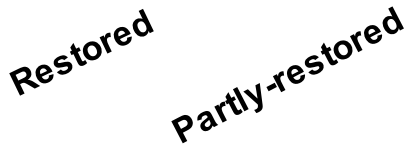




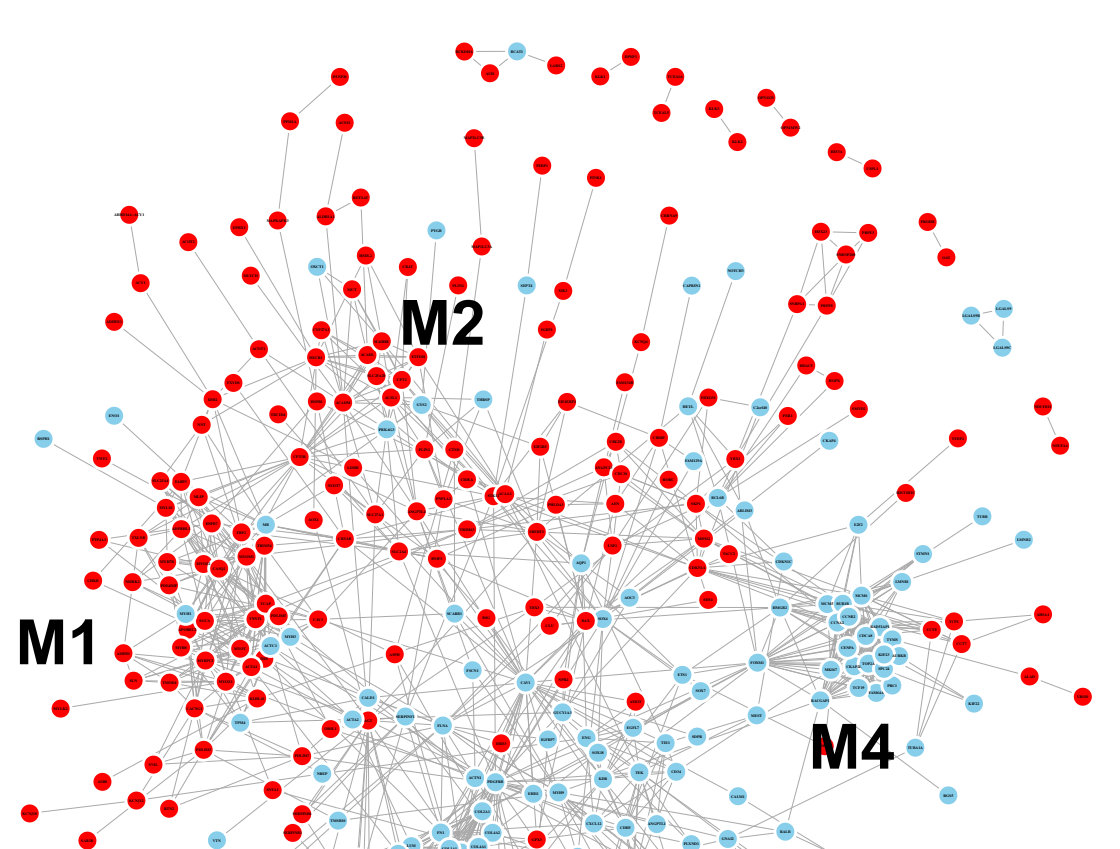

UP in Smn-/-;SMN2

DOWN in Smn-/-;SMN2

M5

b

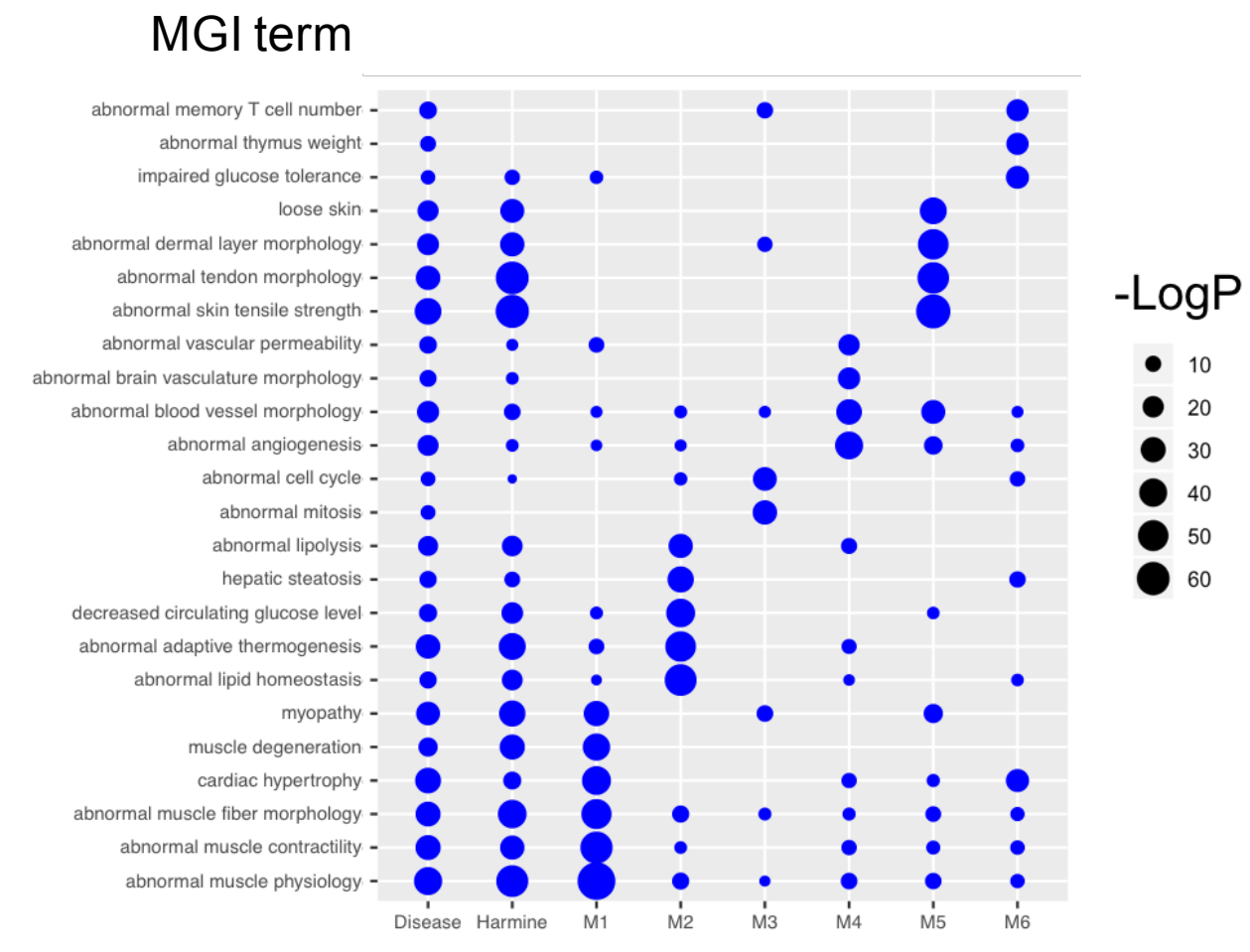

M2

M1

M4.

UP in Harmine treatment

DOWN in Harmine treatment $\mathbf{M 5}^{\circ}$

C

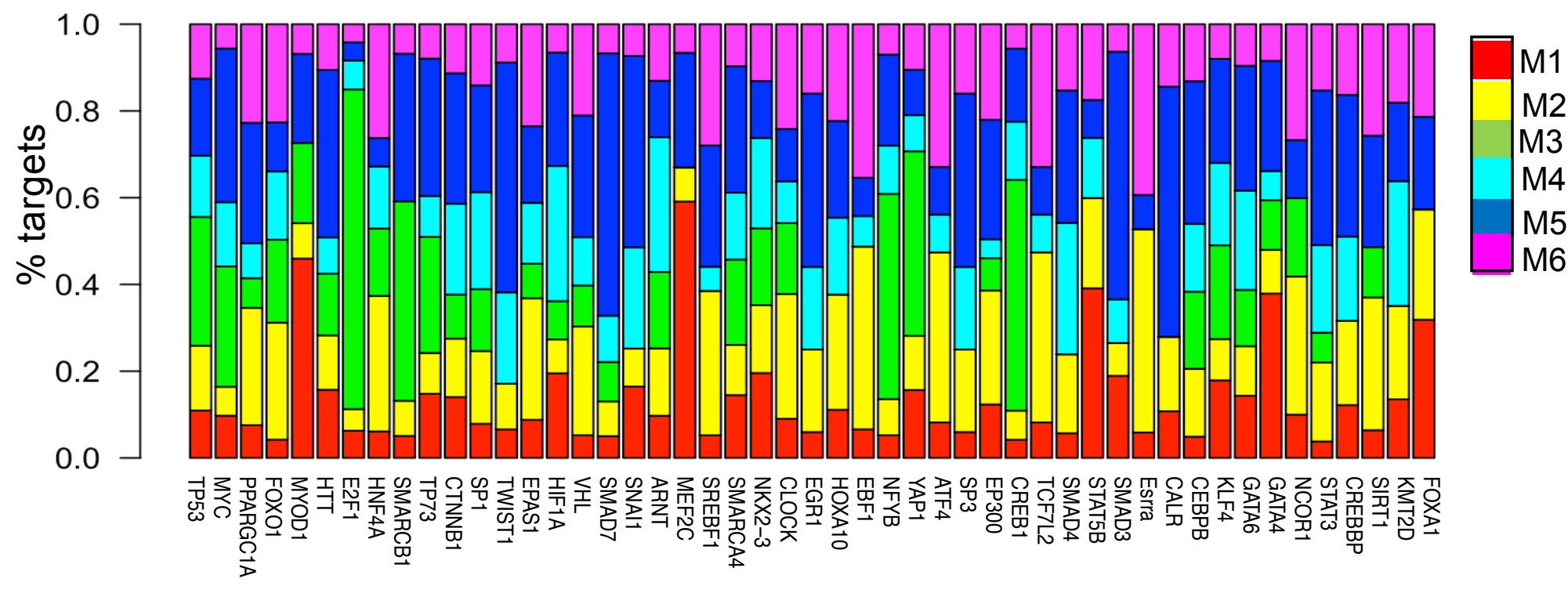



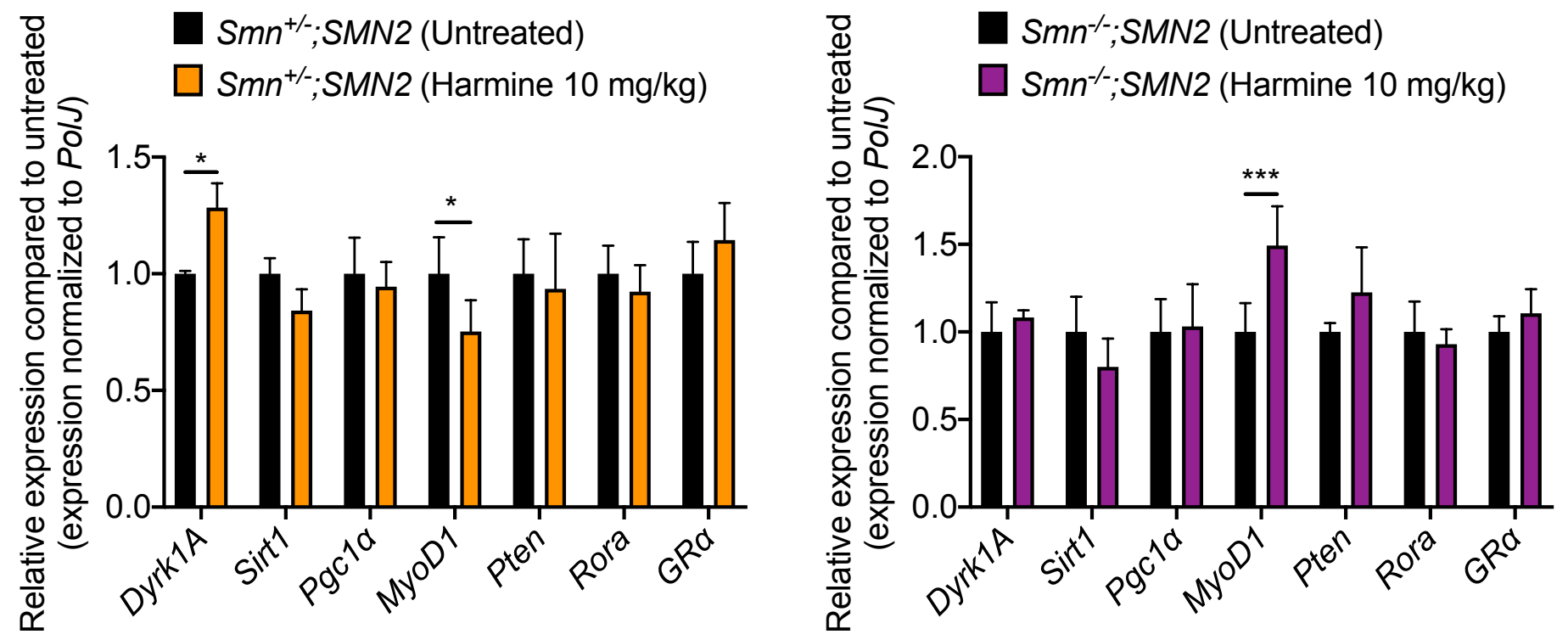

C

Dyrk1A

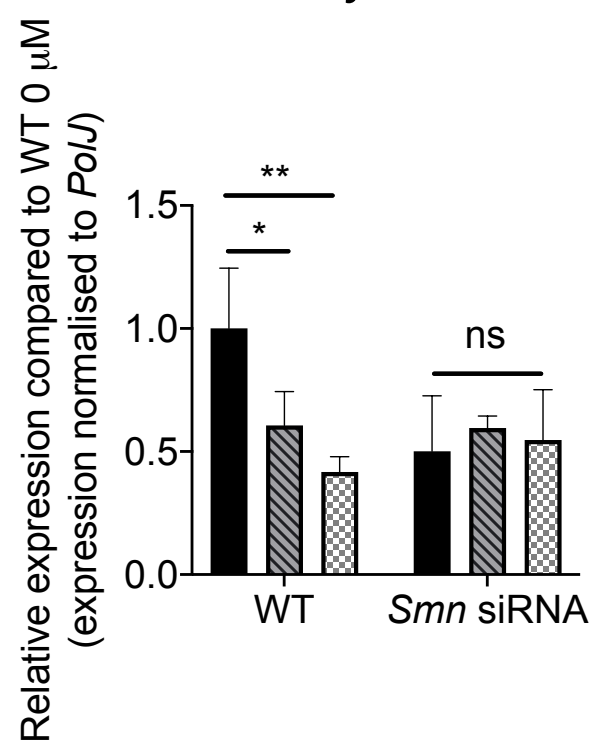

d

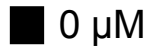

$\mathbb{\mathbb { N }} 25 \mu \mathrm{M}$ 욧 $50 \mathrm{M}$

$\sum_{0}$

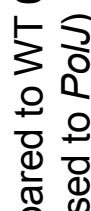

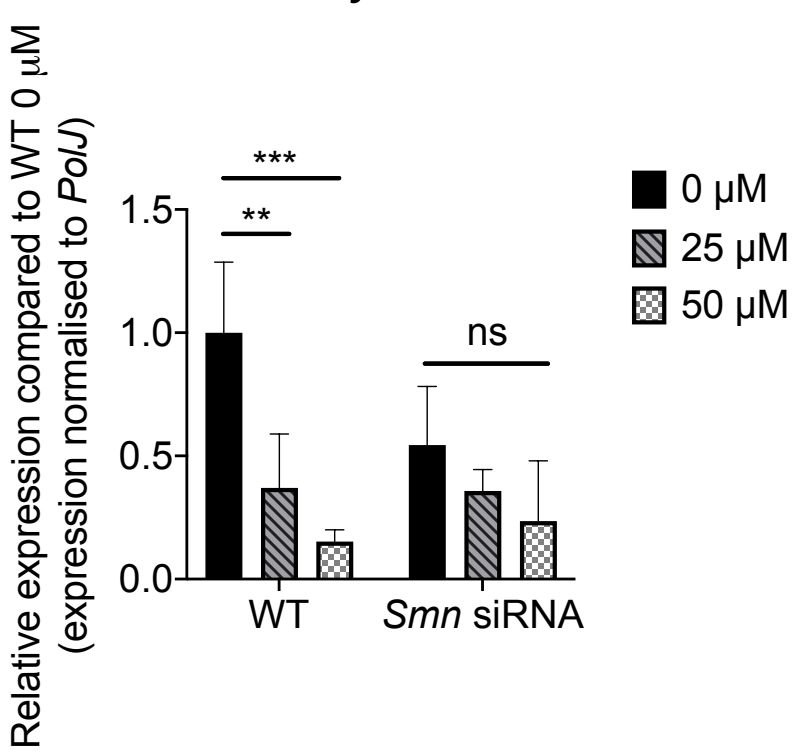

MyoD1 